\title{
Model independent Analysis of Dirac CP Violating Phase for some well known mixing scenarios
}

\author{
Sumit K. GARG ${ }^{a}{ }^{*}$ \\ ${ }^{a}$ Department of Physics, \\ CMR University, Bengaluru 562149, India
}

\begin{abstract}
We present a model independent analysis of Leptonic CP violation for some well known mixing scenarios. In particular, we considered modified schemes for Bimaximal(BM), Democratic(DC), Hexagonal(HG) and Tribimaixmal(TBM) mixing for our numerical investigation. These model independent corrections to mixing matrices are parameterized in terms of complex rotation matrices $(U)$ with related modified PMNS matrix of the forms $\left(U_{i j}^{l} \cdot V_{M}, V_{M} \cdot U_{i j}^{r}\right.$ ) where $U_{i j}^{l, r}$ is complex rotation in ij sector and $V_{M}$ is unperturbed mixing scheme. We present generic formulae for mixing angles, Dirac CP phase $\left(\delta_{C P}\right)$ and Jarkslog Invariant $\left(J_{C P}\right)$ in terms of correction parameters. The parameter space of each modified mixing case is scanned for fitting neutrino mixing angles using $\chi^{2}$ approach and the corresponding predictions for Leptonic CP Phase $\left(\delta_{C P}\right)$ and Jarkslog Invariant $\left(J_{C P}\right)$ has been evaluated from allowed parameter space. The obtained ranges are reported for all viable cases.
\end{abstract}

\footnotetext{
${ }^{*}$ sumit.k@cmr.edu.in
} 


\section{Introduction}

The observation of neutrino oscillations [1] is a major milestone in particle physics over the last few decades. Solar and atmospheric neutrino studies [2, 3] provided the first reliable evidence of neutrino flavor change when these subatomic particles travels through vacuum and matter. These observations undoubtedly confirmed the existence of physics beyond the domain of standard model. With arrival of reactor and accelerator experiments $[4,5,6,7,8]$ in neutrino physics arena, neutrino physics entered into precision era with the determination of oscillation parameters with much greater accuracy. It remarkably helped for improving our understanding about neutrino oscillation physics.

In a 3 flavor scenario, neutrino mixing is described by $3 \times 3$ unitary matrix which can parametrized in terms of 3 mixing angles and 6 phases. However 5 phases are redundant and thus can be rotated away leaving behind only 1 physical phase. Thus light neutrino mixing is given in standard form as [9]

$U=\left(\begin{array}{ccc}1 & 0 & 0 \\ 0 & c_{23} & s_{23} \\ 0 & -s_{23} & c_{23}\end{array}\right)\left(\begin{array}{ccc}c_{13} & 0 & s_{13} e^{-i \delta_{C P}} \\ 0 & 1 & 0 \\ -s_{13} e^{i \delta_{C P}} & 0 & c_{13}\end{array}\right)\left(\begin{array}{ccc}c_{12} & s_{12} & 0 \\ -s_{12} & c_{12} & 0 \\ 0 & 0 & 1\end{array}\right)\left(\begin{array}{ccc}1 & 0 & 0 \\ 0 & e^{i \rho} & 0 \\ 0 & 0 & e^{i \sigma}\end{array}\right)$,

where $c_{i j} \equiv \cos \theta_{i j}, s_{i j} \equiv \sin \theta_{i j}$ and $\delta_{C P}$ is the Dirac CP violating phase. Here two additional phases $\rho$ and $\sigma$ known as Majorana phases are not relevant as they don't affect the neutrino oscillations. Thus we safely assumed their values to be zero in this study. The so far interesting picture which emerged in this scenario is that two mixing angles seems to be large while third one remains small. As far as leptonic CP phase is concerned, the situation is not much clear as still wide range of values $[10,11,12,13,14]$ can be accommodated at $3 \sigma$ level of confidence. Moreover data from long-baseline accelerator, solar and KamLAND is also consistent [14] at $2 \sigma$ or less in CP conserving limit for $\mathrm{NH}$ as well as IH. However on the other hand, some initial hints also emerged from experiments like T2K [15] and NOvA [16] refering towards maximal $\mathrm{CP}$ violation in this sector. Thus neutrino physics is in interesting phase and is suppose to reveal many secrets in forthcoming years.

Before the start of exciting era of non zero $\theta_{13}$, many mixing schemes like tribimaximal [17, 18, 19, 20, 21], bimaximal [22, 23, 24, 25, 26, 27, 28, 29], democratic [30, 31, 32] and Hexagonal [33] were proposed which offered to explain neutrino mixing data with a common novel prediction of zero reactor mixing angle i.e. $\theta_{13}=0$. The atmospheric mixing angle $\left(\theta_{23}\right)$ is maximal for BM, TBM and $\mathrm{HG}$ while it takes a value of of $54.7^{\circ}$ in DC case. The solar mixing angle $\left(\theta_{12}\right)$ is maximal for $\mathrm{BM}$ and $\mathrm{DC}$ scenario while it predicts a lower value of $35.3^{\circ}$ and $30^{\circ}$ in TBM and HG case respectively. However, nuclear reactor based Daya Bay [4] experiment in China which basically looks for disappearance of $\bar{\nu}_{e}$ gave first conclusive result about the fate of $\theta_{13}$. This collaboration reported the value of 1-3 mixing angle consistent 
with data at $5.2 \sigma$ significance level in the range $\sin ^{2} 2 \theta_{13}=0.092 \pm 0.016$ (stat) \pm 0.05 (syst) under a three flavor scenario. Earlier reactor based Japanese experiment, T2K [5] which is a long baseline neutrino oscillation experiment observed similar hints of non zero $\theta_{13}$ corresponding to $\nu_{\mu} \rightarrow \nu_{e}$ transition in a three flavor scenario. The value of 1-3 mixing angle consistent with data at $90 \% \mathrm{CL}$ was reported to be in the range $5^{\circ}\left(5.8^{\circ}\right)<\theta_{13}<16^{\circ}\left(17.8^{\circ}\right)$ for Normal (Inverted) neutrino mass hierarchy. These observations are also being verified by other oscillation experiments like Double Chooz [6], Minos [7] and RENO [8].

The discovery of non zero $\theta_{13}$ is an important turning point in neutrino physics which provided a very crucial input for model building. Thus with these findings along with inputs from recent global fits $[10,11,12,13,14]$ for neutrino masses and mixing angles (given in Table 2), it is quite evident that these mixing scenarios can only provide the main structure of the consistent neutrino matrix at leading order. Hence all such mixing schemes should be tested for possible modifications [34, 35, 36, 37, 38, 39, 40, 41, 42, 43, 44, 45, 46, 47, 48, $49,50,51,52,53,54,55,56,57,58,59,60,61,62,63,64,65,66,67,68,69,70,71,72$, 73, 74, 75, 90, 91, 92, 93, 94, 95, 85, 86, 87, 88, 89, 76, 77, 78, 79, 80, 81, 82, 83, 84] to check their viability with current oscillation data. In literature, these corrections are often being parametrized in terms of complex rotation matrices [90, 91, 92, 93, 94, 95, 96] which acts on 12, 23 or 13 sector of these special matrices. This simpler way of parameterizing the corrections is quite helpful to understand the nature of corrections which a particular sector of these special matrices should get in order to be consistent with neutrino mixing data.

In this study, we addressed the role of possible corrections [99, 100, 97, 98, 96] which are parameterized by one complex rotation matrix [96] for these mixing schemes. Thus modified PMNS matrix will be of the forms $\left(U_{i j} \cdot V_{M}, V_{M} \cdot U_{i j}\right)$, where $V_{M}$ is unmodified mixing matrix and $U_{i j}$ is rotation in $i j$ sector of complex plane. As we know from theoretical point of view, the form of PMNS matrix is given by $U_{P M N S}=U_{l}^{\dagger} U_{\nu}$ so these modifications might originate from charged lepton $[101,102,103,104,105,106,107,108,109,110,111]$ and neutrino $[112,113,114,115,116,117,118,119]$ sector. Here we performed numerical analysis by scanning parameter space for each case of these mixing schemes. The main characteristics of our detailed numerical investigation are:

(i) The latest global fit results [14] have been used for our model independent analysis. These results are obtained by taking into account latest data from long-baseline accelerator, solar and Kam-LAND, short-baseline reactor, and atmospheric neutrino experiments.

(ii) We invoked $\chi^{2}$ approach $[97,98,96]$ for studying the situation of mixing angle fitting in parameter space. This will give essential information about magnitude and sign of correction parameters. It will also help in comparing level of fitting achieved for various cases under different modification schemes.

(iii) The correlations among neutrino mixing angles are studied by varying all mixing angles 
in their $3 \sigma$ limits. It is different from the approach where one out of three mixing angles is fixed at a particular value for discussing the correlation between remaining two mixing angles. This in our view show a complete picture and thus we present our numerical findings in terms of 2 dimensional scatter plots instead of going for line plots.

(iv) Finally the limits on leptonic CP phase $\left(\delta_{C P}\right)$ and Jarkslog invaraint $\left(J_{C P}\right)$ has been derived from the parameter space which is consistent with 3 mixing angles global fit data. The obtained ranges will thus act as a prediction of that mixing case.

These results can be helpful for understanding the structure of corrections that these well known mixing schemes require in order to be consistent with neutrino mixing data. This model independent investigation can also be useful in filtering out viable models from vast number of possibilities in neutrino model building physics. Moreover all allowed cases have clear prediction of CP Dirac phase which can be easily tested from current/planned neutrino experiments. However mapping these results from model dependent prospective is deferred for future consideration.

The paper is organized as follows. In section 2, we give general discussion about methodology of our work. In sections 3-5, we present our numerical results for various possible correction cases under different mixing schemes. Finally in section 6 , we give the summary and conclusions of our study.

\section{General Setup}

The form of mixing matrix for mixing scenarios under consideration is given as follows:

$$
\begin{aligned}
U_{\mathrm{TBM}}= & \left(\begin{array}{rrr}
\sqrt{\frac{2}{3}} & \sqrt{\frac{1}{3}} & 0 \\
-\sqrt{\frac{1}{6}} & \sqrt{\frac{1}{3}} & \sqrt{\frac{1}{2}} \\
-\sqrt{\frac{1}{6}} & \sqrt{\frac{1}{3}} & -\sqrt{\frac{1}{2}}
\end{array}\right), U_{\mathrm{BM}}=\left(\begin{array}{rrr}
\sqrt{\frac{1}{2}} & \sqrt{\frac{1}{2}} & 0 \\
-\frac{1}{2} & \frac{1}{2} & \sqrt{\frac{1}{2}} \\
\frac{1}{2} & -\frac{1}{2} & \sqrt{\frac{1}{2}}
\end{array}\right), \\
U_{\mathrm{DC}} & =\left(\begin{array}{rrr}
\sqrt{\frac{1}{2}} & \sqrt{\frac{1}{2}} & 0 \\
\sqrt{\frac{1}{6}} & -\sqrt{\frac{1}{6}} & -\sqrt{\frac{2}{3}} \\
-\sqrt{\frac{1}{3}} & \sqrt{\frac{1}{3}} & -\sqrt{\frac{1}{3}}
\end{array}\right), U_{\mathrm{HM}}=\left(\begin{array}{ccc}
\frac{1}{2} & 0 \\
-\frac{1}{2 \sqrt{2}} & \frac{\sqrt{3}}{2 \sqrt{2}} & -\sqrt{\frac{1}{2}} \\
-\frac{1}{2 \sqrt{2}} & \frac{\sqrt{3}}{2 \sqrt{2}} & \sqrt{\frac{1}{2}}
\end{array}\right) .
\end{aligned}
$$




\begin{tabular}{lcccc}
\hline Mixing Angle & BM mixing & DC mixing & TBM mixing & HG mixing \\
\hline$\theta_{23}^{\circ}$ & 45 & 54.7 & 45 & 45 \\
\hline$\theta_{12}^{\circ}$ & 45 & 45 & 35.3 & 30 \\
\hline$\theta_{13}^{\circ}$ & 0 & 0 & 0 & 0 \\
\hline
\end{tabular}

Table 1: Mixing angle values from unpertrubed special matrices. All angles are in degrees $\left(\theta^{\circ}\right)$.

\begin{tabular}{|c|c|c|c|c|}
\hline Normal Hierarchy & Best fit & $1 \sigma$ range & $2 \sigma$ range & $3 \sigma$ range \\
\hline $\sin ^{2} \theta_{12} / 10^{-1}$ & 3.04 & $2.91-3.18$ & $2.78-3.32$ & $2.65-3.46$ \\
\hline $\sin ^{2} \theta_{13} / 10^{-2}$ & 2.14 & $2.07-2.23$ & $1.98-2.31$ & $1.90-2.39$ \\
\hline $\sin ^{2} \theta_{23} / 10^{-1}$ & 5.51 & $4.81-5.70$ & $4.48-5.88$ & $4.30-6.02$ \\
\hline \multicolumn{5}{|l|}{$\begin{array}{l}\text { Inverted Hierar- } \\
\text { chy }\end{array}$} \\
\hline $\sin ^{2} \theta_{12} / 10^{-1}$ & 3.03 & $2.90-3.17$ & $2.77-3.31$ & $2.64-3.45$ \\
\hline $\sin ^{2} \theta_{13} / 10^{-2}$ & 2.18 & $2.11-2.26$ & $2.02-2.35$ & $1.95-2.43$ \\
\hline $\sin ^{2} \theta_{23} / 10^{-1}$ & 5.57 & $5.33-5.74$ & $4.86-5.89$ & $4.44-6.03$ \\
\hline
\end{tabular}

Table 2: Three-flavor oscillation neutrino mixing angles from fit to global data [14].

The resulting value of mixing angles from above mixing schemes are given in Table 1. They share a common prediction of vanishing value of reactor mixing angle i.e. $\theta_{13}=0^{\circ}$. The atmospheric mixing angle $\left(\theta_{23}\right)$ is $45^{\circ}$ for $\mathrm{BM}, \mathrm{TBM}$ and $\mathrm{HG}$ case while it takes a higher value of $54.7^{\circ}$ in $\mathrm{DC}$ case. However solar mixing angle $\left(\theta_{12}\right)$ is $45^{\circ}$ for $\mathrm{BM}$ and $\mathrm{DC}$ scenario while it takes a value of $35.3^{\circ}$ and $30^{\circ}$ in TBM and HG case respectively. Thus these mixing angles are in conflict with recent experimental observations which provide best fit values at $\theta_{13} \sim 8^{\circ}, \theta_{12} \sim 33^{\circ}$ and $\theta_{23} \sim 47^{\circ}$. Hence these mixing schemes should be probed for possible corrections in order to investigate their consistency with current neutrino oscillation data.

In this study, we investigated different cases pertaining to PMNS matrix of the forms $U_{X} \cdot V_{M}$ and $V_{M} \cdot U_{X}$ where $U_{X}$ denotes a complex rotation in $i j$ sector and $V_{M}$ is any one of these special matrices. The correction matrix $U_{X}$ can be expressed in terms of mixing matrix as $R_{X}=\left\{R_{23}, R_{13}, R_{12}\right\}$ in general with a single phase parameter $(\sigma)$ as follows

$$
U_{12}=\left(\begin{array}{ccc}
\cos \alpha & \sin \alpha e^{-i \sigma} & 0 \\
-\sin \alpha e^{i \sigma} & \cos \alpha & 0 \\
0 & 0 & 1
\end{array}\right), U_{23}=\left(\begin{array}{ccc}
1 & 0 & 0 \\
0 & \cos \beta & \sin \beta e^{-i \sigma} \\
0 & -\sin \beta e^{i \sigma} & \cos \beta
\end{array}\right)
$$




$$
U_{13}=\left(\begin{array}{ccc}
\cos \gamma & 0 & \sin \gamma e^{-i \sigma} \\
0 & 1 & 0 \\
-\sin \gamma e^{i \sigma} & 0 & \cos \gamma
\end{array}\right)
$$

Here $R_{12}, R_{23}$ and $R_{13}$ represent the rotations in 12, 23 and 13 sector with corresponding rotation angle $\alpha, \beta, \gamma$ respectively. The related PMNS matrix for single rotation case is given by:

$$
\begin{aligned}
& V_{\mathrm{ij}}^{L}=U_{i j}^{l} \cdot V_{M}, \\
& V_{\mathrm{ij}}^{R}=V_{M} \cdot U_{i j}^{r},
\end{aligned}
$$

where $(i j)=(12),(13),(23)$ respectively.

The effect of these corrections is studied by implementing $\chi^{2}$ function which is a measure of overall departure of obtained values of mixing angles in parameter space from the values that are given from global fits $[10,11,12,13,14]$. It is given by the expression

$$
\chi^{2}=\sum_{i=1}^{3}\left\{\frac{\theta_{i}(P)-\theta_{i}^{e x p}}{\delta \theta_{i}^{\exp }}\right\}^{2}
$$

with $\theta_{i}(P)$ are the values of mixing angles obtained from mixing scheme in parameter space which is a function of one of correction parameter $(\alpha, \beta, \gamma)$ along with a phase parameter $\sigma$. $\theta_{i}^{e x p}$ are the best fitted value of neutrino mixing angles obtained from latest global fit data with corresponding $1 \sigma$ deviation $\delta \theta_{i}$. The unperturbed values of $\chi^{2}$ for all considered mixing schemes with Normal and Inverted Hierarchy are given in Table 6.

\begin{tabular}{|l||l|l|l|l|}
\hline \multicolumn{5}{|c|}{$\chi^{2}$ value with uncorrected Mixing schemes } \\
\hline Hierarchy & BM & DC & TBM & HG \\
\hline Normal & 927.6 & 933.1 & 721.5 & 732.8 \\
\hline Inverted & 1065.5 & 1086.4 & 857.6 & 868.0 \\
\hline
\end{tabular}

As evident from above table, $\mathrm{NH}$ is little favorable as its $\chi^{2}$ value is lower as compared to its IH counterpart. Here we investigated the role of various possible mixing cases for bringing $\chi^{2}$ further down in parameter space and thus reaching closer to experimental best fit. A good numerical fit should produce low $\chi^{2}$ value in parameter region.

\section{$3 \quad$ Numerical Results}

In this section, we present and discuss numerical findings of our investigation for Normal and Inverted Hierarchy. We studied the role of these corrected mixing schemes in producing 
large $\theta_{13}[34,35,36,37,38,39,40,41,42,43,44,45,46,47,48,49,50,51,52,53,54,55$, $56,57,58,59,60,61,62,63$ and fitting other mixing angles. The resulting value of $\delta_{C P}$ and $J_{C P}$ from allowed parameter space is treated as prediction of that mixing case. All these well known mixing scenarios have common prediction of $\theta_{13}=0$ since 13 element is zero. Thus all such mixing schemes can be put in following generic form

$$
V_{\text {Mix }}=\left(\begin{array}{rrr}
a_{11} & a_{12} & 0 \\
a_{21} & a_{22} & a_{23} \\
a_{31} & a_{32} & a_{33}
\end{array}\right)
$$

where all matrix elements are real for our considered cases. Here we present our formulae for mixing angles, $\delta_{C P}$ and $J_{C P}$ in terms of correction parameters using above generic form. The same formuale can also be used in other situations which have similar prediction of $\theta_{13}=0$.

The scanning of parameter space is performed by randomly varying $\alpha, \beta$ and $\gamma$ in the range $[-0.5,0.5]$ whereas the phase parameter $(\sigma)$ is chosen from the interval $[-\pi, \pi]$. A good numerical fit to global fitting data should produce low $\chi^{2}$ and thus plotting data points are selected by invoking the condition $\chi^{2}<\chi_{i}^{2}(i=\mathrm{BM}, \mathrm{DC}, \mathrm{TBM}$ and HG) in our scanning subroutines. The discussion on obtained results is presented from following plots:

(i) A 2-dimensional projection of $\chi^{2}$ over correction parameters revealing overall situation of fitting in parameter space. The reported value of $\chi_{\min }^{2}$ in these plots corresponds to best level of fitting for all three mixing angles.

(ii) Scattered plot of $\theta_{13}$ over $\theta_{23}-\theta_{12}$ plane for getting information about best level of fitting and ranges of mixing angles that can be achieved in parameter space.

(iii) The scattered plots of $\delta_{C P}$ and $J_{C P}$ vs mixing angles within allowed region for determining the range of these quantities.

In figures of $\chi^{2}$ over modification parameters $\left(\theta_{1}, \theta_{2}\right)$ red, blue and light green color regions pertains to $\chi^{2}$ value in the interval $[0,3],[3,10]$ and $>10$ respectively. The white region of plot corresponds to completely disallowed part of $\chi^{2}>\chi_{\text {unperturbed }}^{2}$.

In neutrino mixing angles figures, green band refers to $1 \sigma$ and full colored band to $3 \sigma$ values of $\theta_{13}$. Also ' $\times$ ' refers to the case which is unable to fit mixing angles even at $3 \sigma$ level while '-' pertains to the situation where $\theta_{13}$ remains unchanged i.e. $\theta_{13}=0$. For showing the mapping between left and right figures, we marked the $\chi^{2}<3,[3,10]$ regions in mixing angle plots using color codings. The white region corresponds to $3<\chi^{2}<10$ whereas yellow region refers to $\chi^{2}<3$. Horizontal and vertical dashed black, dashed pink and thick black lines corresponds to $1 \sigma, 2 \sigma$ and $3 \sigma$ ranges of the other two mixing angles. Now we will take up all considered cases one by one. 


\section{Rotations- $U_{i j}^{l} . V_{M}$}

Here we first consider the corrections for which the form of modified PMNS matrix is given by $U_{P M N S}=U_{i j}^{l} \cdot V_{M}$. It will introduce changes in $i^{\text {th }}$ and $j^{\text {th }}$ row of unperturbed matrix. In subsequent subsections, we investigate the role of this mixing scheme in fitting neutrino mixing angles and its prediction for $\delta_{C P}$ and $J_{C P}$.

\subsection{Rotation}

This mixing scheme pertains to complex rotation in 12 sector of these special matrices. Here rotation matrix operates from left side and thus impart changes in first two rows of unperturbed mixing matrix. The expressions for neutrino mixing angles in this scheme are given as

$$
\begin{aligned}
\sin ^{2} \theta_{13} & =a_{23}^{2} \sin ^{2} \alpha \\
\sin ^{2} \theta_{23} & =\frac{a_{23}^{2} \cos ^{2} \alpha}{\cos ^{2} \theta_{13}}, \\
\sin ^{2} \theta_{12} & =\frac{a_{12}^{2} \cos ^{2} \alpha+a_{22}^{2} \sin ^{2} \alpha+a_{12} a_{22} \sin 2 \alpha \cos \sigma}{\cos ^{2} \theta_{13}}
\end{aligned}
$$

The Jarsklog invariant and CP Dirac phase is given by the expressions

$$
\begin{aligned}
\sin ^{2} \delta_{C P} & =C_{12 L}^{2}\left(\frac{p_{1 \alpha}}{p_{2 \alpha \sigma} p_{3 \alpha \sigma}}\right) \sin ^{2} \sigma, \\
J_{C P} & =J_{12 L} \sin 2 \alpha \sin \sigma
\end{aligned}
$$

where

$$
\begin{aligned}
J_{12 L} & =\frac{1}{2} a_{23}^{2} \sqrt{1-a_{23}^{2}} C_{12 L}, \\
C_{12 L} & =\frac{\left(a_{11} a_{22}-a_{12} a_{21}\right)\left(a_{11} a_{12}+a_{21} a_{22}\right)}{a_{23}^{2} \sqrt{1-a_{23}^{2}}} \\
p_{1 \alpha} & =1+a_{23}^{4} \sin ^{4} \alpha-2 a_{23}^{2} \sin ^{2} \alpha, \\
p_{2 \alpha \sigma} & =1-a_{12}^{2} \cos ^{2} \alpha-\left(a_{22}^{2}+a_{23}^{2}\right) \sin ^{2} \alpha-a_{12} a_{22} \cos \sigma \sin 2 \alpha, \\
p_{3 \alpha \sigma} & =a_{12}^{2} \cos ^{2} \alpha+a_{22}^{2} \sin ^{2} \alpha+a_{12} a_{22} \cos \sigma \sin 2 \alpha
\end{aligned}
$$

Fig. 1-5 show the numerical results corresponding to this mixing case for normal hierarchy $(\mathrm{NH})$. The notable features of this mixing are: 
(i) Here $\theta_{23}$ remain close to its unperturbed value since it receives corrections of $O\left(\theta^{2}\right)$ from parameter $\alpha$. Since for DC case unperturbed $\theta_{23} \sim 54.7$ so it is disfavored completely in this mixing scheme.

(ii) As fitting of $\theta_{13}$ and $\theta_{23}$ is only governed by $\alpha$ so its allowed range is much constrained in parameter space. e.g. for TBM case, the fitting of $\theta_{13}$ under its $3 \sigma$ domain constraints the magnitude of correction parameter $|\alpha| \in[0.1962(0.1988), 0.2204(0.2223)]$ which in turn fixes $\theta_{23} \in\left[44.29^{\circ}\left(44.28^{\circ}\right), 44.44^{\circ}\left(44.43^{\circ}\right)\right]$ for corresponding $\alpha$ values in $\mathrm{NH}(\mathrm{IH})$ case. However $\theta_{12}$ possess much wider range of values since it receives corrections from $\alpha$ as well from phase parameter $\sigma$.

(iii) The minimum value of $\chi^{2} \sim 20.2(30.2), 51.4(70.4), 1.95(11.0)$ and 1.94(11.0) for BM, DC, TBM and HG respectively with $\mathrm{NH}(\mathrm{IH})$ case. Here BM barely manages to fit all mixing angles in $3 \sigma$ level for a very minute region of parameter space while DC is not viable. However HG and TBM are much favorable as they are able to fit all mixing angles within $1 \sigma$ level in NH case. Since $1 \sigma$ range for $\theta_{23}$ in IH is quite constrained as compared to its NH counterpart so fitted value of $\theta_{23}$ in TBM and $\mathrm{HG}$ goes outside its $1 \sigma$ range. Thus this case is only allowed at $2 \sigma$ level.

(iv) Leptonic phase $\delta_{C P}$ lies in the range $-4.4(-4.7) \leq \delta_{C P} \leq 3.4(5.3)$ for BM while it is confined in 39.0(40.4) $\leq\left|\delta_{C P}\right| \leq 78.7(79.2)$ for modified HG and $61.0(60.9) \leq\left|\delta_{C P}\right| \leq 89.9(89.9)$ corrected TBM matrix.

(v) The Jarkslog invariant $\left(J_{C P}\right)$ remains in range $-0.0027(-0.0029) \leq J_{C P} \leq 0.0021(0.0033)$ for BM while it is confined in $0.020(0.021) \leq\left|J_{C P}\right| \leq 0.032(0.032)$ for corrected HG while it remains $0.026 \leq\left|J_{C P}\right| \leq 0.035$ for TBM mixing matrix.

\begin{tabular}{|l||l|l|l|l|l|l|}
\hline \multicolumn{7}{|c|}{ Best fit with Mixing data } \\
\hline Rotation & $\chi_{\min }^{2}$ & $\theta_{12}^{\circ}$ & $\theta_{23}^{\circ}$ & $\theta_{13}^{\circ}$ & $\left|\delta_{C P}^{\circ}\right|$ & $\left|J_{C P}\right|$ \\
\hline BM & $20.2(30.2)$ & $36.02(35.97)$ & $44.3(44.2)$ & $8.86(8.92)$ & $0.498(1.888)$ & $0.0003(0.0011)$ \\
\hline DC & $51.4(70.4)$ & $\mathbf{3 8 . 9}(\mathbf{3 8 . 8})$ & $\mathbf{5 4 . 2 ( 5 4 . 2 )}$ & $8.54(8.62)$ & $0.152(1.879)$ & $0.00008(0.0011)$ \\
\hline TBM & $1.95(11.0)$ & $33.37(33.39)$ & $44.3(44.3)$ & $8.42(8.48)$ & $80.55(80.85)$ & $0.032(0.032)$ \\
\hline HG & $1.94(11.0)$ & $33.51(33.38)$ & $44.3(44.3)$ & $8.41(8.48)$ & $61.89(62.98)$ & $0.029(0.029)$ \\
\hline
\end{tabular}

Table 3: Neutrino Mixing angles, $\left|\delta_{C P}^{\circ}\right|$ and $\left|J_{C P}\right|$ corresponding to $\chi_{\min }^{2}$ numerical fit. The mixing angle value that lies outside its best fit $3 \sigma$ range is marked in boldface.

\subsection{Rotation}

This case refers to rotation in 13 sector of these special matrices that bring modifications in Ist and 3rd row of unperturbed mixing matrix. The expressions of neutrino mixing angles 

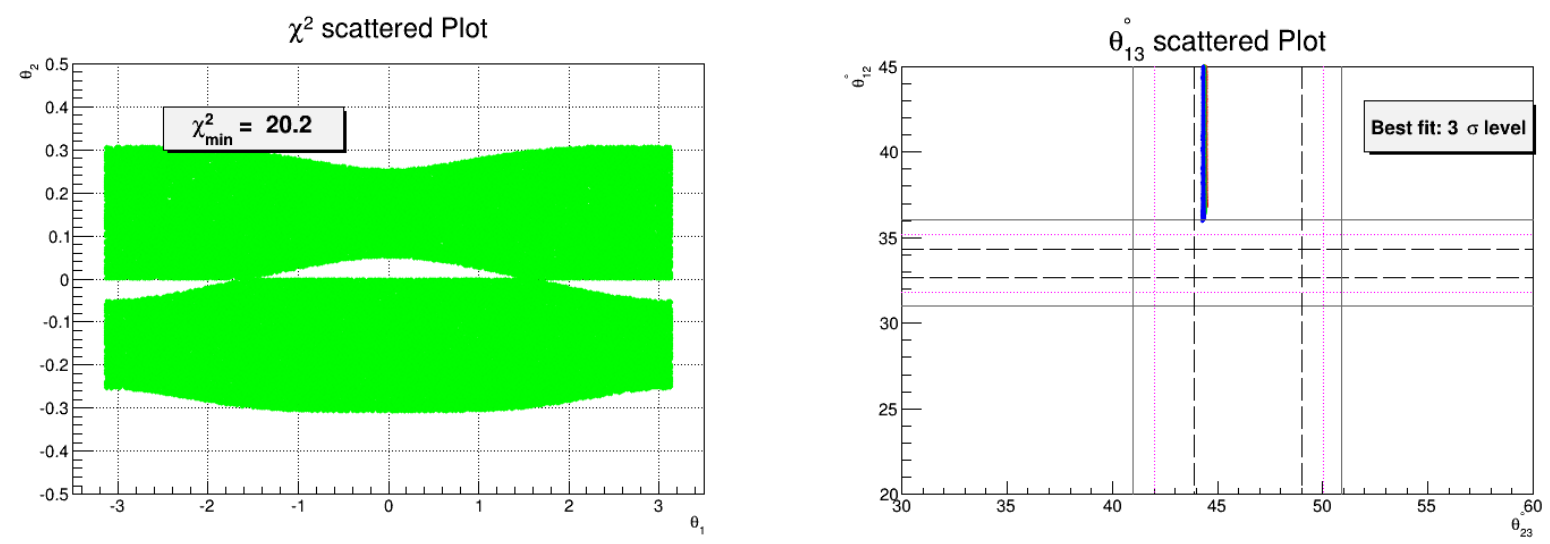

Figure 1: Scattered plot of $\chi^{2}$ (left fig.) over $\alpha-\sigma$ plane and $\theta_{13}$ (right fig.) over $\theta_{23}-\theta_{12}$ (in degrees) plane for $U_{12}^{B M L}$ rotation scheme. The information about other color coding and various horizontal, vertical lines in right fig. is given in text.
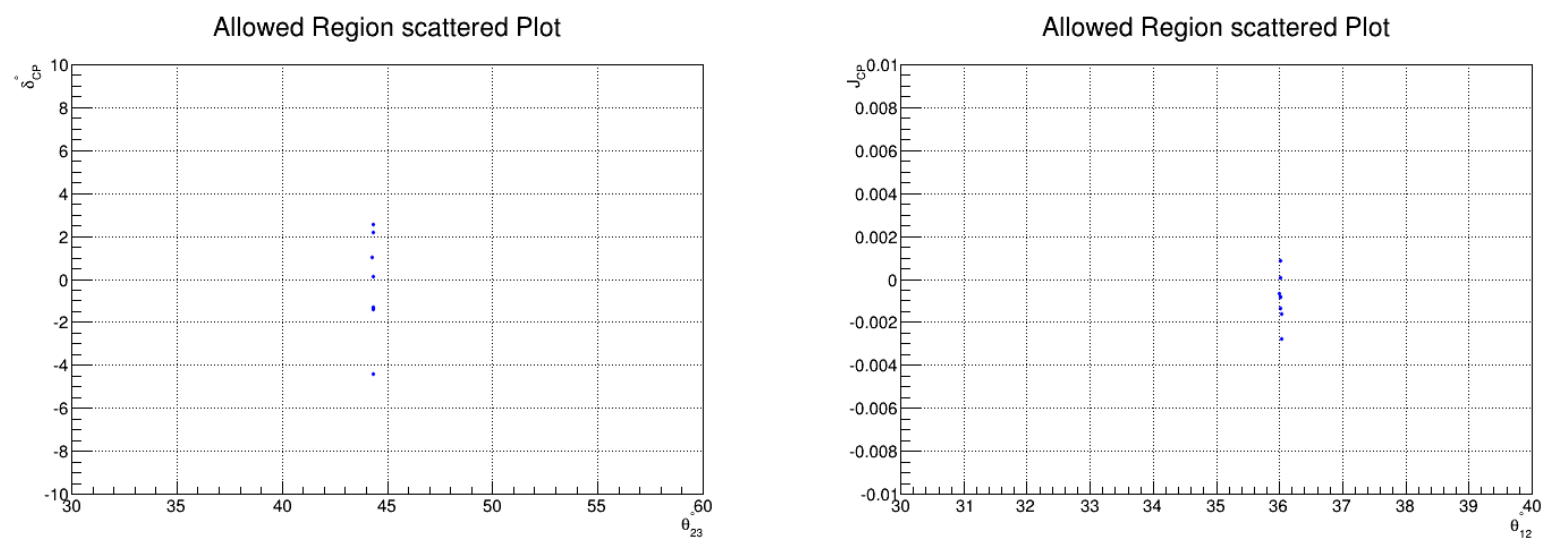

Figure 2: Scattered plot of $\delta_{C P}$ (left fig.) vs $\theta_{23}$ (in degrees) and scattered plot of $J_{C P}$ (right fig.) over $\theta_{12}$ (in degrees) plane for $U_{12}^{B M L}$ rotation scheme.

for this case are given as

$$
\begin{aligned}
\sin ^{2} \theta_{13} & =a_{33}^{2} \sin ^{2} \gamma \\
\sin ^{2} \theta_{23} & =\frac{a_{23}^{2}}{\cos ^{2} \theta_{13}}, \\
\sin ^{2} \theta_{12} & =\frac{a_{12}^{2} \cos ^{2} \gamma+a_{32}^{2} \sin ^{2} \gamma+a_{12} a_{32} \sin 2 \gamma \cos \sigma}{\cos ^{2} \theta_{13}} \\
\sin ^{2} \delta_{C P} & =C_{13 L}^{2}\left(\frac{p_{1 \gamma}}{p_{2 \gamma} p_{3 \gamma \sigma} p_{4 \gamma \sigma}}\right) \cos ^{2} \gamma \sin ^{2} \sigma,
\end{aligned}
$$



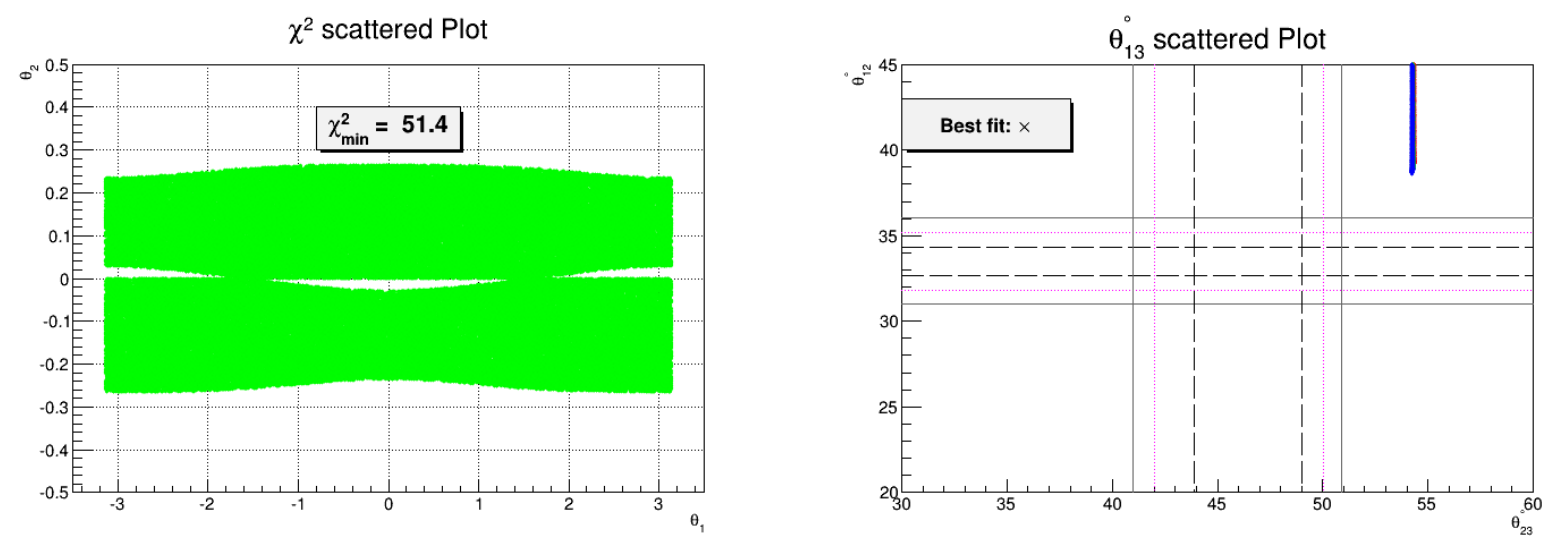

Figure 3: Scattered plot of $\chi^{2}$ (left fig.) over $\alpha-\sigma$ plane and $\theta_{13}$ (right fig.) over $\theta_{23}-\theta_{12}$ (in degrees) plane for $U_{12}^{D C L}$ rotation scheme. The information about other color coding and various horizontal, vertical lines in right fig. is given in text.
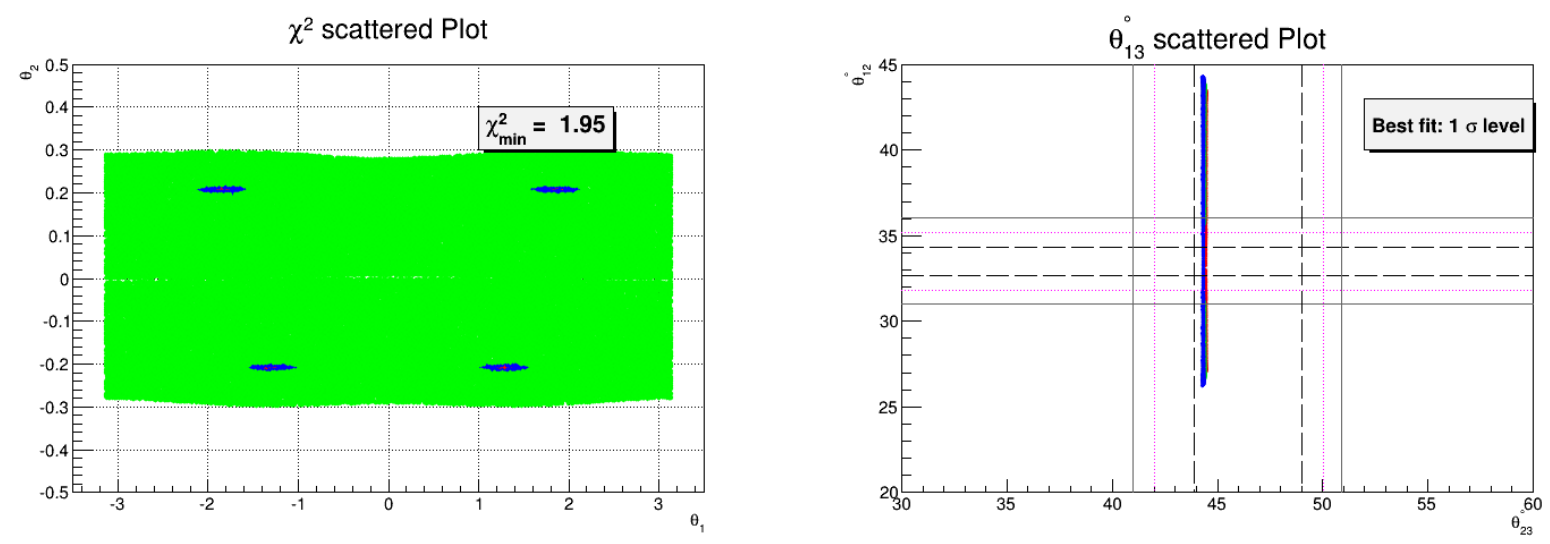

Figure 4: Scattered plot of $\chi^{2}$ (left fig.) over $\alpha-\sigma$ plane and $\theta_{13}$ (right fig.) over $\theta_{23}-\theta_{12}$ (in degrees) plane for $U_{12}^{T B M L}$ rotation scheme.

$$
J_{C P}=J_{13 L} \sin 2 \gamma \sin \sigma
$$

where

$$
\begin{aligned}
J_{13 L} & =\frac{1}{2} a_{23} a_{33} C_{13 L}, \\
C_{13 L} & =\frac{a_{21} a_{22}}{a_{23} a_{33}}\left(a_{11} a_{32}-a_{12} a_{31}\right), \\
p_{1 \gamma} & =1+a_{33}^{4} \sin ^{4} \gamma-2 a_{33}^{2} \sin ^{2} \gamma, \\
p_{2 \gamma} & =1-a_{23}^{2}-a_{33}^{2} \sin ^{2} \gamma \\
p_{3 \gamma \sigma} & =1-a_{12}^{2} \cos ^{2} \gamma-\left(a_{32}^{2}+a_{33}^{2}\right) \sin ^{2} \gamma-a_{12} a_{32} \cos \sigma \sin 2 \gamma, \\
p_{4 \gamma \sigma} & =a_{12}^{2} \cos ^{2} \gamma+a_{32}^{2} \sin ^{2} \gamma+a_{12} a_{32} \cos \sigma \sin 2 \gamma
\end{aligned}
$$



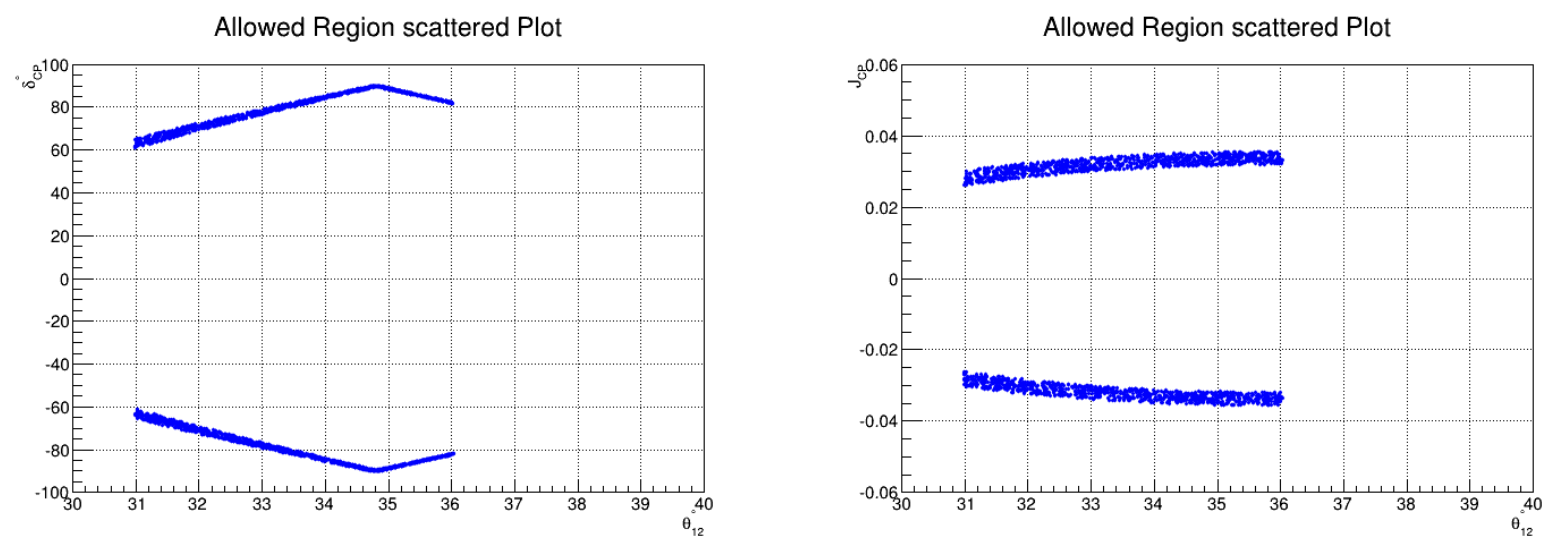

Figure 5: Scattered plot of $\delta_{C P}$ (left fig.) vs $\theta_{12}$ (in degrees) and scattered plot of $J_{C P}$ (right fig.) over $\theta_{12}$ (in degrees) plane for $U_{12}^{T B M L}$ rotation scheme.
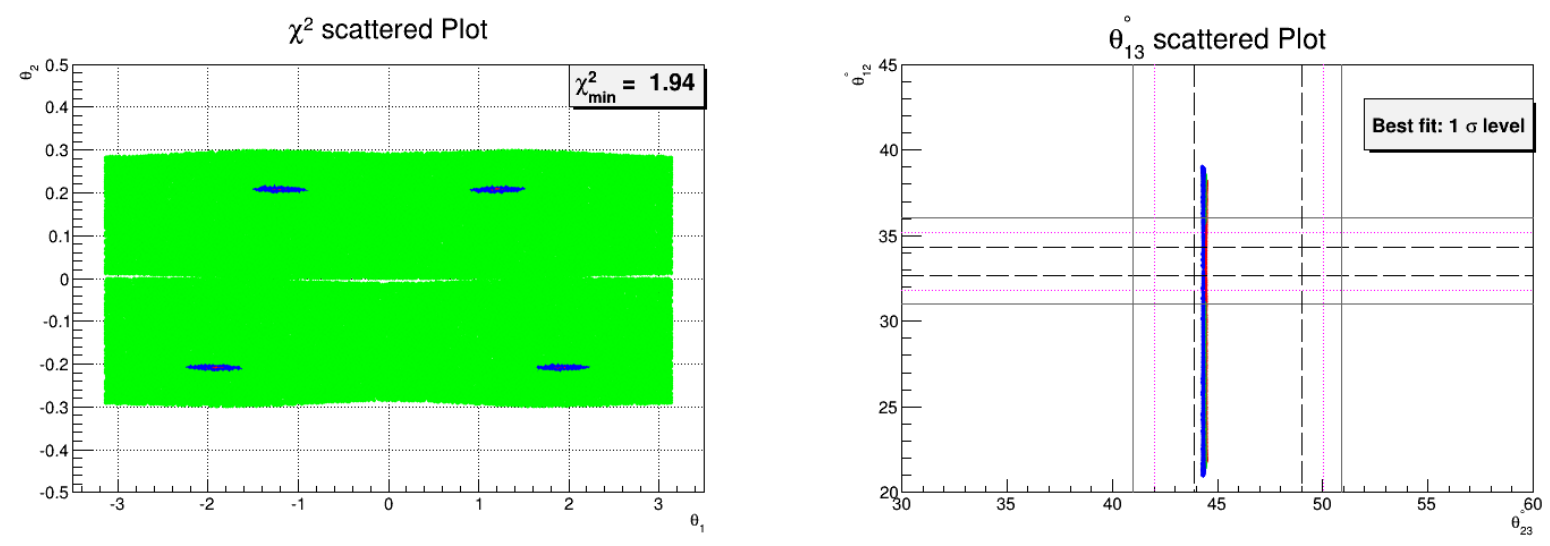

Figure 6: Scattered plot of $\chi^{2}$ (left fig.) over $\alpha-\sigma$ plane and $\theta_{13}$ (right fig.) over $\theta_{23}-\theta_{12}$ (in degrees) plane for $U_{12}^{H G L}$ rotation scheme.

Fig. 8-12 show the numerical results corresponding to our considered HG mixing. The main features of this perturbative scheme are:

(i) Like previous case, $\theta_{23}$ remains very close to its unperturbed value as corrections to this angle enters through $\theta_{13}$. Thus DC case is disfavored completely.

(ii) The numerical fitting of $\theta_{13}$ and $\theta_{23}$ is only governed by $\alpha$ so its allowed range is much restricted in parameter space. e.g. for TBM case, the fitting of $\theta_{13}$ under its $3 \sigma$ domain constraints the magnitude of correction parameter $|\alpha| \in[0.1962(0.1988), 0.2204(0.2223)]$ which in turn fixes $\theta_{23} \in\left[45.55^{\circ}\left(45.56^{\circ}\right), 45.70^{\circ}\left(45.71^{\circ}\right)\right]$ for corresponding $\gamma$ values with $\mathrm{NH}(\mathrm{IH})$ case. However $\theta_{12}$ possess much wider range of values since it receives corrections from $\alpha$ as well from phase parameter $\sigma$.

(iii) The minimum value of $\chi^{2} \sim 18.9(23.4), 8.56(36.8), 0.82(5.0)$ and $0.81(5.0)$ for BM, DC, TBM and HG respectively with $\mathrm{NH}(\mathrm{IH})$ case. Here also BM barely manages to fit all 

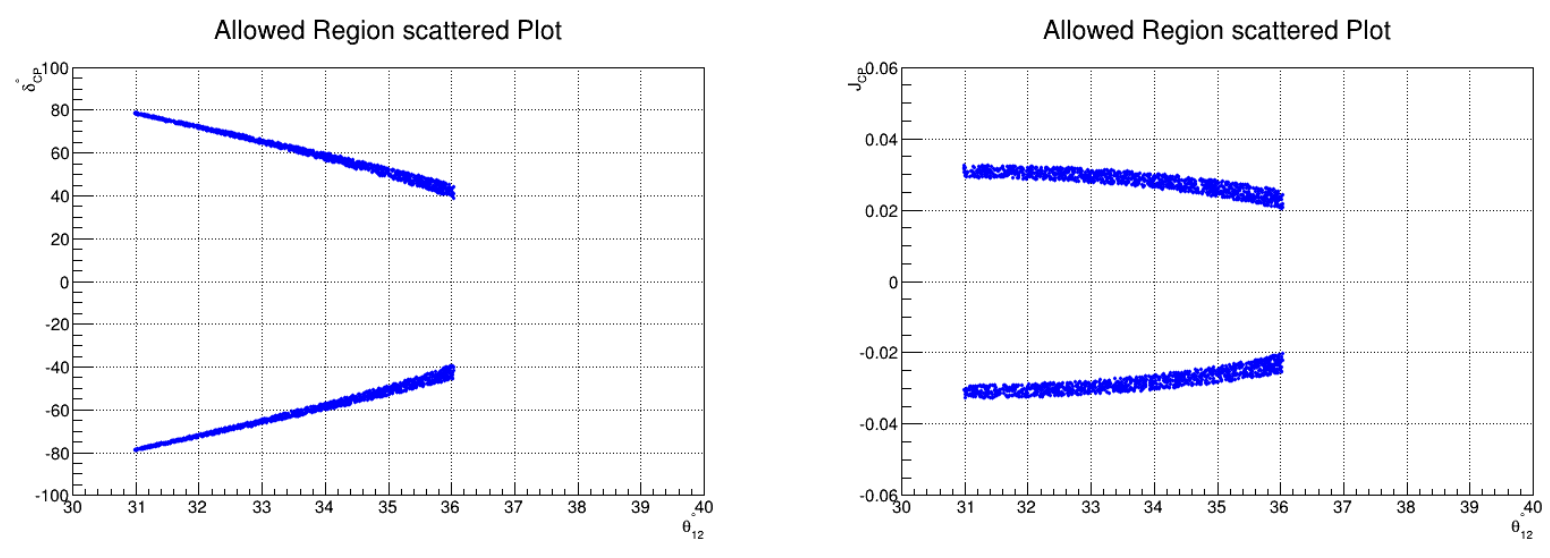

Figure 7: Scattered plot of $\delta_{C P}$ (left fig.) vs $\theta_{12}$ (in degrees) and scattered plot of $J_{C P}$ (right fig.) over $\theta_{12}$ (in degrees) plane for $U_{12}^{H G L}$ rotation scheme.

mixing angles in $3 \sigma$ level for a very minute region of parameter space while DC is not viable. However HG and TBM are much favorable as they are able to fit all mixing angles within $1 \sigma$ level for $\mathrm{NH}$. However for $\mathrm{IH}, \theta_{23}$ stays outside its $1 \sigma$ range so it is only allowed at $2 \sigma$ level.

(iv) Leptonic phase $\delta_{C P}$ lies in the range $-3.6(-4.9) \leq \delta_{C P} \leq 4.2(5.5)$ for BM while it is confined in 39.0(40.4) $\leq\left|\delta_{C P}\right| \leq 78.7(79.2)$ for modified HG and 61.0(60.9) $\leq\left|\delta_{C P}\right| \leq 89.9(89.9)$ corrected TBM matrix.

(v) The Jarkslog invariant $\left(J_{C P}\right)$ remains in range $-0.0025(-0.0034) \leq J_{C P} \leq 0.0026(0.0030)$ for BM while it is confined in $0.020(0.021) \leq\left|J_{C P}\right| \leq 0.032(0.032)$ for corrected $\mathrm{HG}$ and $0.026 \leq\left|J_{C P}\right| \leq 0.035$ TBM mixing matrix.

\begin{tabular}{|l||l|l|l|l|l|l|}
\hline \multicolumn{7}{|c|}{ Best Fit with Mixing data } \\
\hline Rotation & $\chi_{\min }^{2}$ & $\theta_{12}^{\circ}$ & $\theta_{23}^{\circ}$ & $\theta_{13}^{\circ}$ & $\left|\delta_{C P}^{\circ}\right|$ & $\left|J_{C P}\right|$ \\
\hline BM & $18.9(23.4)$ & $36.03(35.96)$ & $45.6(45.7)$ & $8.86(8.92)$ & $0.594(0.302)$ & $0.0003(0.0001)$ \\
\hline DC & $8.56(36.8)$ & $33.42(33.35)$ & $\mathbf{5 5 . 6}(\mathbf{5 5 . 6})$ & $8.41(8.46)$ & $17.51(17.62)$ & $0.009(0.009)$ \\
\hline TBM & $0.82(5.0)$ & $33.37(33.41)$ & $45.6(45.6)$ & $8.42(8.49)$ & $80.55(81.0)$ & $0.032(0.032)$ \\
\hline HG & $0.81(5.0)$ & $33.51(33.39)$ & $45.6(45.6)$ & $8.41(8.48)$ & $61.89(62.95)$ & $0.029(0.029)$ \\
\hline
\end{tabular}

Table 4: Neutrino Mixing angles, $\left|\delta_{C P}^{\circ}\right|$ and $\left|J_{C P}\right|$ corresponding to $\chi_{\min }^{2}$ numerical fit. The mixing angle value that lies outside its best fit $3 \sigma$ range is marked in boldface. 

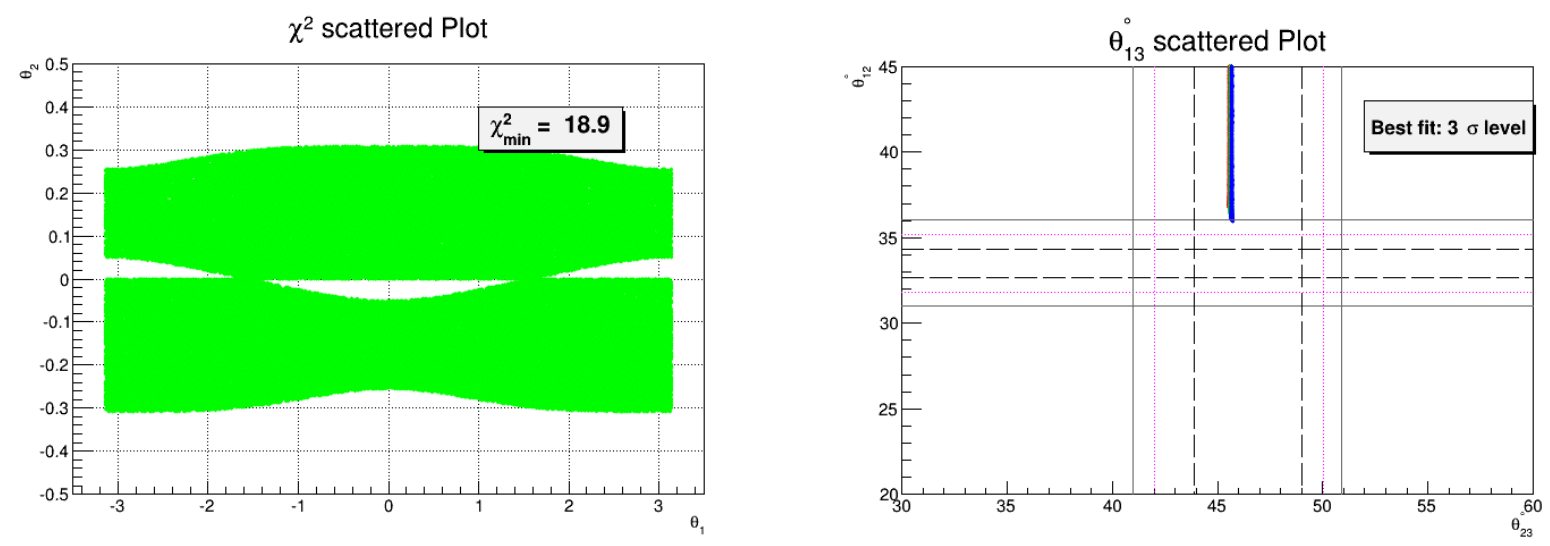

Figure 8: Scattered plot of $\chi^{2}$ (left fig.) over $\gamma-\sigma$ plane and $\theta_{13}$ (right fig.) over $\theta_{23}-\theta_{12}$ (in degrees) plane for $U_{13}^{B M L}$ rotation scheme.
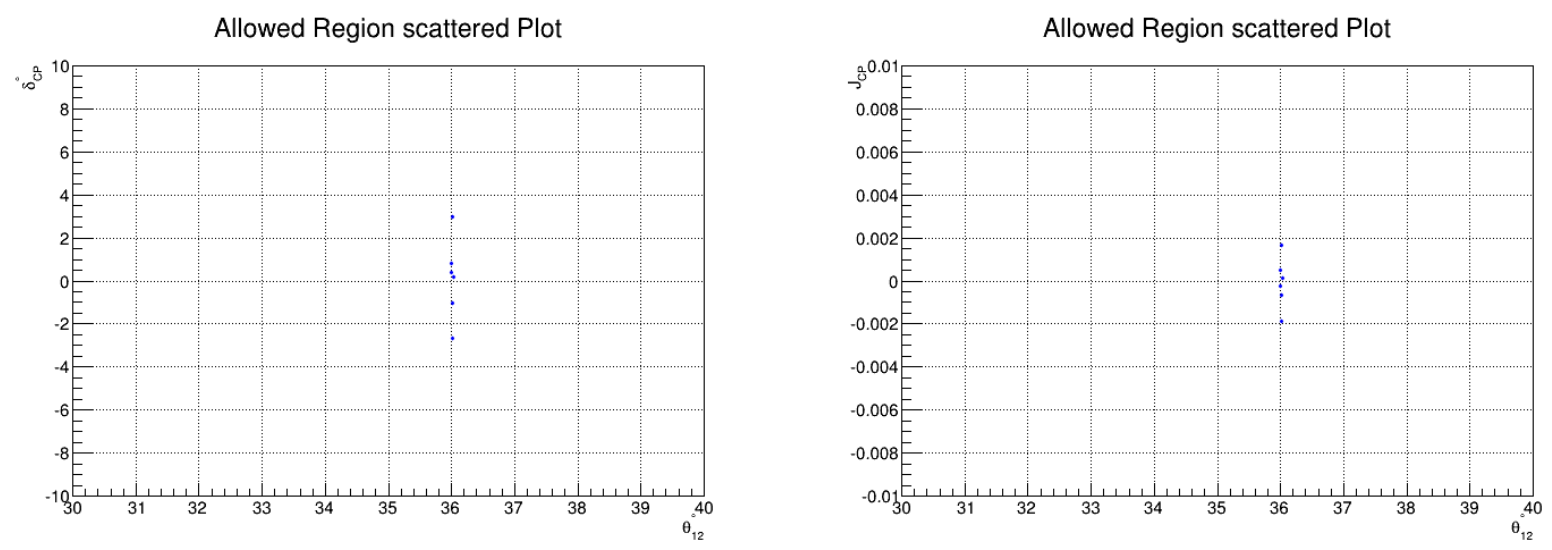

Figure 9: Scattered plot of $\delta_{C P}$ (left fig.) vs $\theta_{12}$ (in degrees) and scattered plot of $J_{C P}$ (right fig.) over $\theta_{12}$ (in degrees) plane for $U_{13}^{B M L}$ rotation scheme.

\subsection{Rotation}

Here rotation matrix imparts corrections in last two rows of unperturbed matrix. Thus reactor mixing angle, $\theta_{13}$ doesn't receive any corrections in this scheme. Thus we left this case without going for any further discussion.

\section{$5 \quad$ Rotations- $V_{M} \cdot U_{i j}^{r}$}

Here we take up the modifications for which PMNS matrix is given by $U_{P M N S}=V_{M} \cdot U_{i j}^{r}$. This scheme will introduce changes in $i^{\text {th }}$ and $j^{\text {th }}$ column of unperturbed mixing matrix. We will investigate the role of these perturbations in fitting the neutrino mixing angles and its prediction for Dirac CP Phase $\left(\delta_{C P}\right)$. 

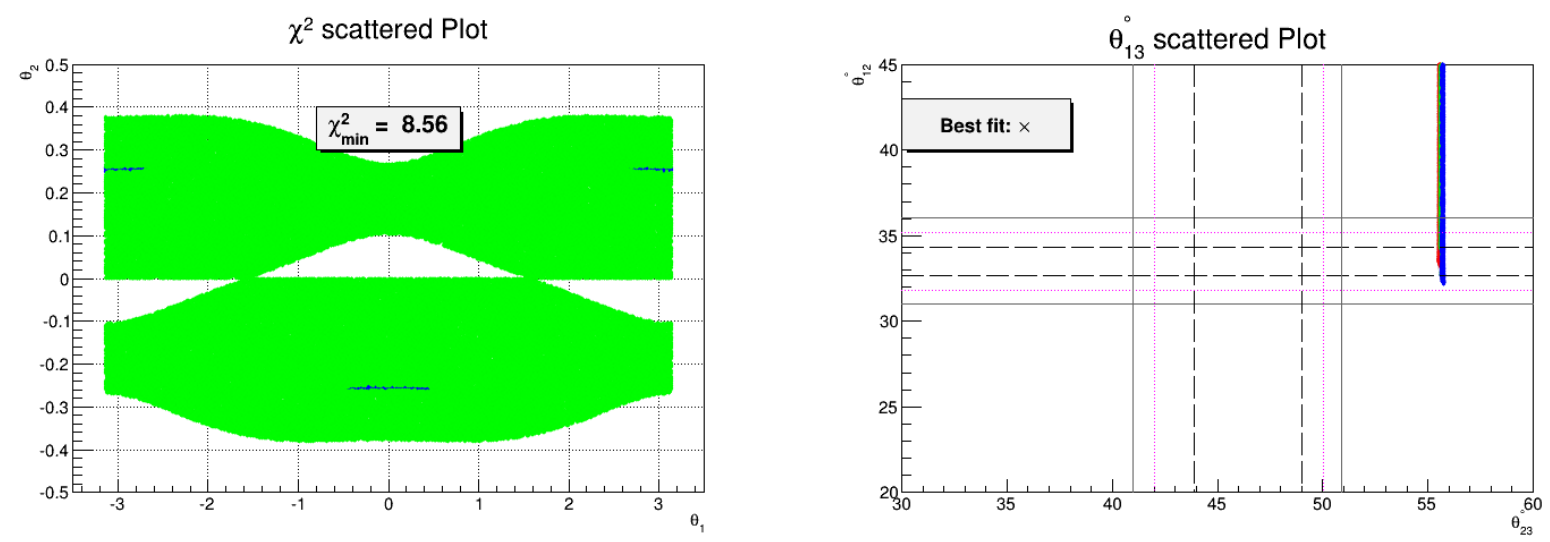

Figure 10: Scattered plot of $\chi^{2}$ (left fig.) over $\gamma-\sigma$ plane and $\theta_{13}$ (right fig.) over $\theta_{23}-\theta_{12}$ (in degrees) plane for $U_{13}^{D C L}$ rotation scheme.
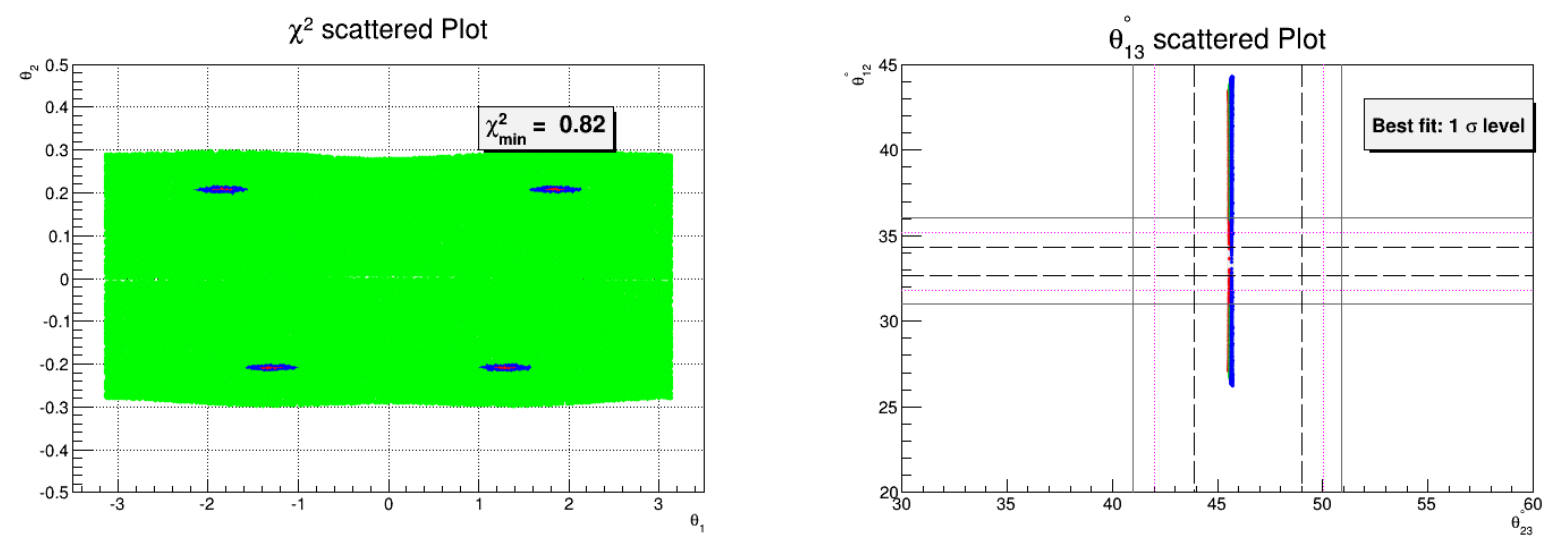

Figure 11: Scattered plot of $\chi^{2}$ (left fig.) over $\gamma-\sigma$ plane and $\theta_{13}$ (right fig.) over $\theta_{23}-\theta_{12}$ (in degrees) plane for $U_{13}^{T B M L}$ rotation scheme.

\section{$5.1 \quad 12$ Rotation}

In this case, rotation matrix imparts corrections in first two columns of unperturbed matrix. Thus reactor mixing angle, $\theta_{13}$ doesn't get any modifications in this scheme. Hence this case is not of significance and we left it for any further discussion.

\subsection{Rotation}

This case corresponds to rotation in 13 sector of these special matrices. The expressions for mixing angles in this case are given as

$$
\sin ^{2} \theta_{13}=a_{11}^{2} \sin ^{2} \gamma
$$


Allowed Region scattered Plot

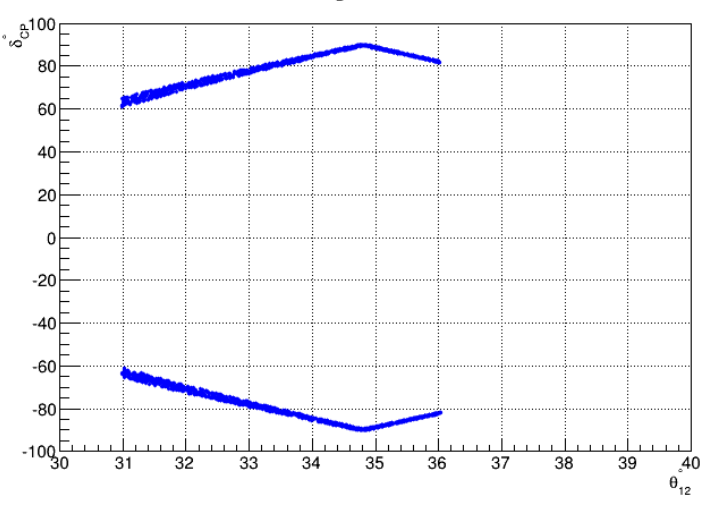

Allowed Region scattered Plot

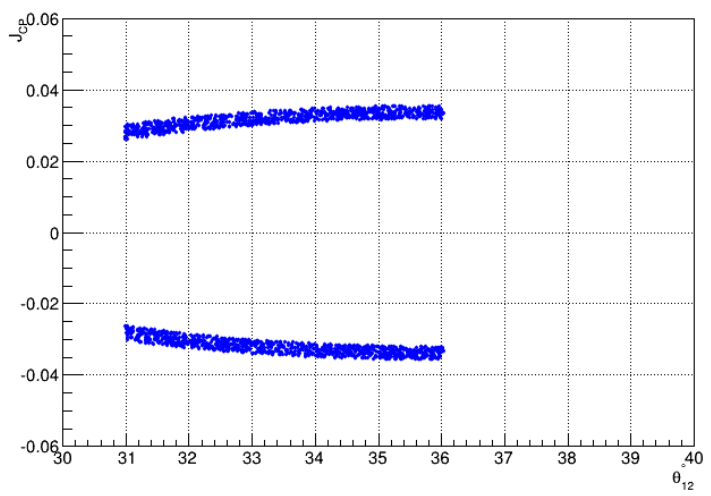

Figure 12: Scattered plot of $\delta_{C P}$ (left fig.) vs $\theta_{12}$ (in degrees) and scattered plot of $J_{C P}$ (right fig.) over $\theta_{12}$ (in degrees) plane for $U_{13}^{T B M L}$ rotation scheme.
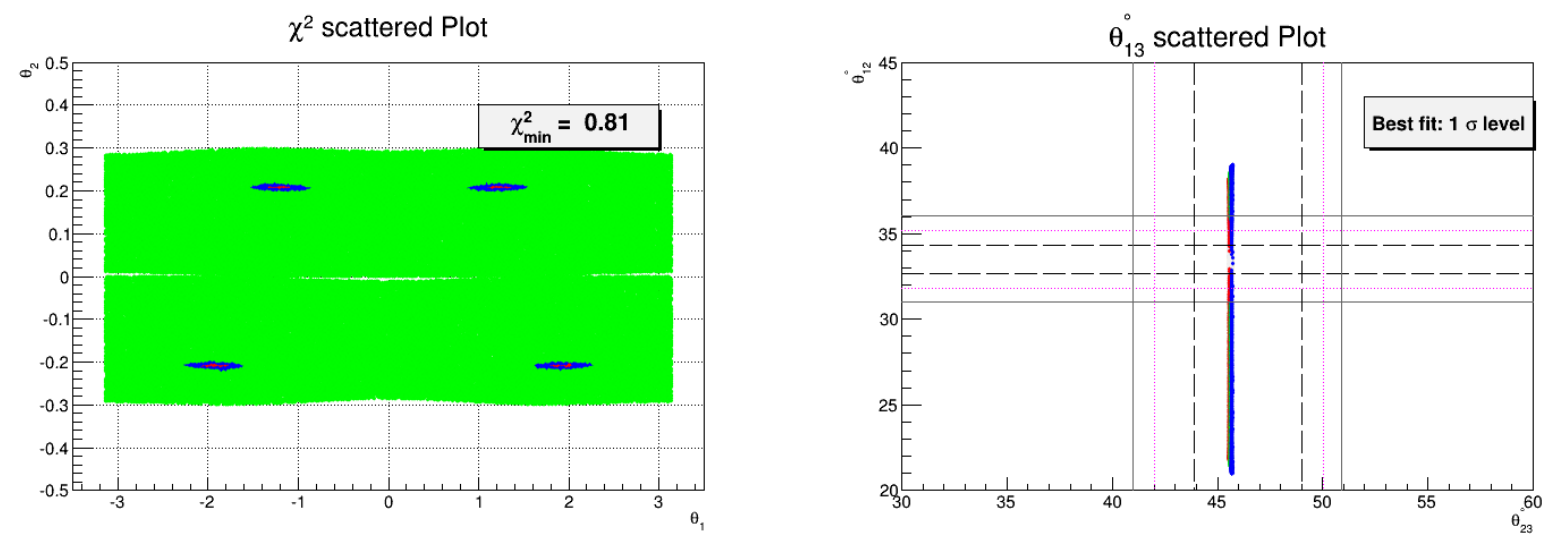

Figure 13: Scattered plot of $\chi^{2}$ (left fig.) over $\gamma-\sigma$ plane and $\theta_{13}$ (right fig.) over $\theta_{23}-\theta_{12}$ (in degrees) plane for $U_{13}^{H G L}$ rotation scheme.

$$
\begin{aligned}
\sin ^{2} \theta_{12} & =\frac{a_{12}^{2}}{\cos ^{2} \theta_{13}}, \\
\sin ^{2} \theta_{23} & =\frac{a_{23}^{2} \cos ^{2} \gamma+a_{21}^{2} \sin ^{2} \gamma+a_{21} a_{23} \sin 2 \gamma \cos \sigma}{\cos ^{2} \theta_{13}}
\end{aligned}
$$

The Jarsklog invariant and CP Dirac Phase is given by expressions

$$
\begin{aligned}
\sin ^{2} \delta_{C P} & =C_{13 R}^{2}\left(\frac{p_{1 \gamma}}{p_{2 \gamma} p_{3 \gamma \sigma} p_{4 \gamma \sigma}}\right) \cos ^{2} \gamma \sin ^{2} \sigma \\
J_{C P} & =J_{13 R} \sin 2 \gamma \sin \sigma
\end{aligned}
$$

where 

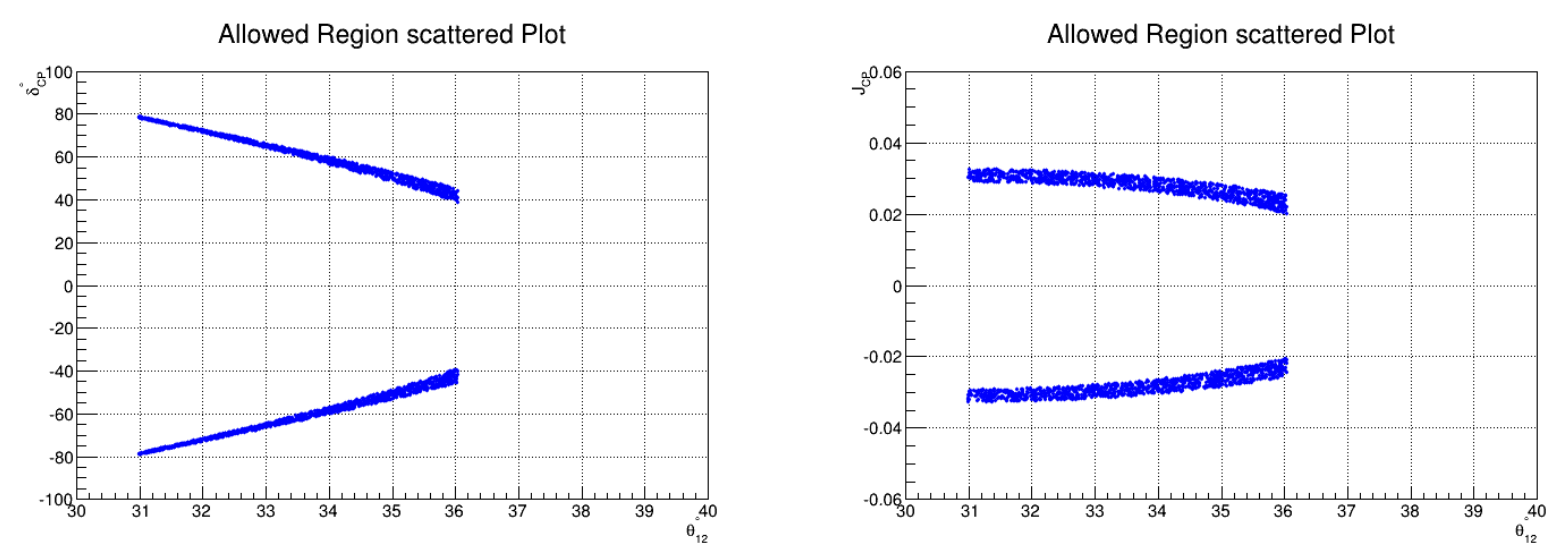

Figure 14: Scattered plot of $\delta_{C P}$ (left fig.) vs $\theta_{12}$ (in degrees) and scattered plot of $J_{C P}$ (right fig.) over $\theta_{12}$ (in degrees) plane for $U_{13}^{H G L}$ rotation scheme.

$$
\begin{aligned}
C_{13 R} & =a_{22} a_{23}, \\
J_{13 R} & =\frac{1}{2} a_{11} a_{12} C_{13 R}, \\
p_{1 \gamma} & =1+a_{11}^{4} \sin ^{4} \gamma-2 a_{11}^{2} \sin ^{2} \gamma, \\
p_{2 \gamma} & =1-a_{12}^{2}-a_{11}^{2} \sin ^{2} \gamma, \\
p_{3 \gamma \sigma} & =1-a_{23}^{2} \cos ^{2} \gamma-\left(a_{11}^{2}+a_{21}^{2}\right) \sin ^{2} \gamma-a_{21} a_{23} \cos \sigma \sin 2 \gamma, \\
p_{4 \gamma \sigma} & =a_{23}^{2} \cos ^{2} \gamma+a_{21}^{2} \sin ^{2} \gamma+a_{21} a_{23} \cos \sigma \sin 2 \gamma
\end{aligned}
$$

Fig. 15-18 show the numerical results corresponding to perturbed HG case. The main features of these corrections are given as:

(i) Here solar mixing angle $\left(\theta_{12}\right)$ receives very minor corrections through $\sin \theta_{13}$ and thus its value remain close to its original prediction. Thus BM and DC will be disfavored for this scheme.

(ii) As fitting of $\theta_{13}$ and $\theta_{12}$ is only governed by $\gamma$ so its allowed range is much constrained in parameter space. e.g. for TBM case, the fitting of $\theta_{13}$ under its $3 \sigma$ domain constraints the magnitude of correction parameter $|\gamma| \in[0.1696(0.1719), 0.1905(0.1921)]$ which in turn fixes $\theta_{12} \in\left[35.65^{\circ}\left(35.66^{\circ}\right), 35.75^{\circ}\left(35.76^{\circ}\right)\right]$ for corresponding $\gamma$ values. However $\theta_{12}$ possess much wider range of values since it receives corrections from $\alpha$ as well from phase parameter $\sigma$. (iii) The minimum value of $\chi^{2} \sim 234.7(237.5), 234.7(237.5), 7.35(7.82)$ and $12.9(12.3)$ for BM, DC, TBM and HG respectively with $\mathrm{NH}(\mathrm{IH})$ case. Here only TBM manages to fit all mixing angles within $3 \sigma$ level. The corrected HG predicts low value of $\theta_{12}$ which is outside its $3 \sigma$ range and hence it is not viable. 

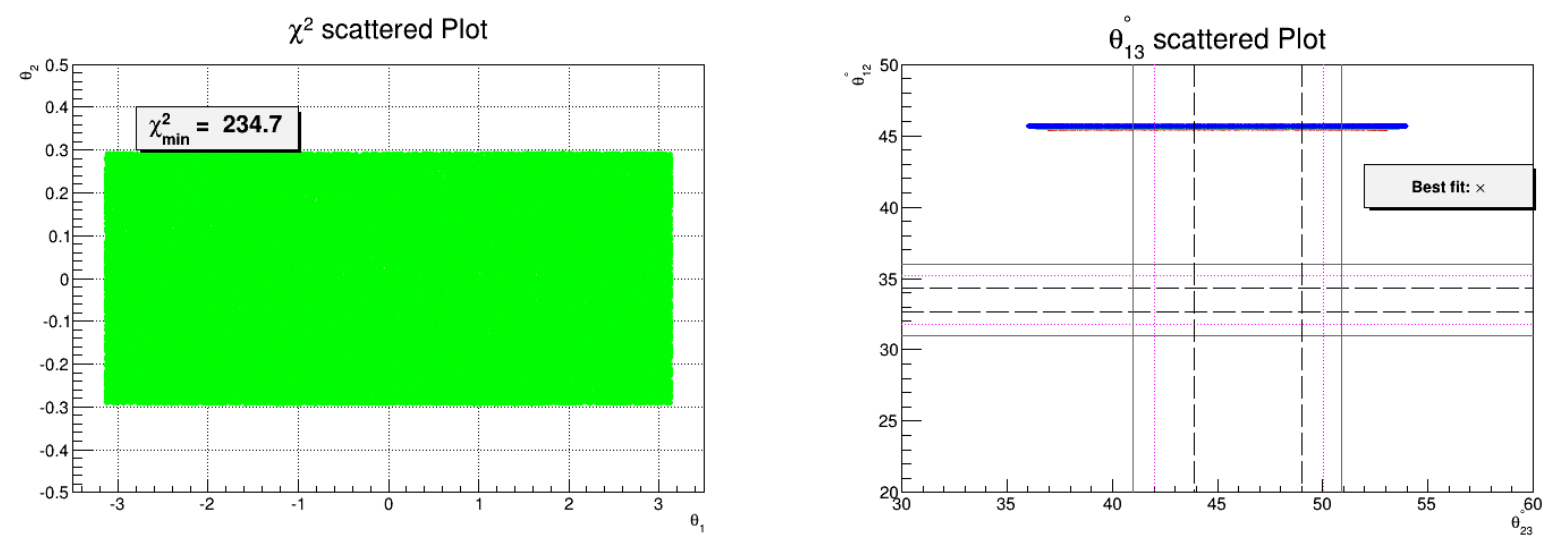

Figure 15: Scattered plot of $\chi^{2}$ (left fig.) over $\gamma-\sigma$ plane and $\theta_{13}$ (right fig.) over $\theta_{23}-\theta_{12}$ (in degrees) plane for $U_{13}^{B M R}$ rotation scheme.

(iv) Leptonic phase $\delta_{C P}$ lies in the range $0 \leq\left|\delta_{C P}\right| \leq 89.9$ while $J_{C P}$ in the range $0(0) \leq$ $\left|J_{C P}\right| \leq 0.0357(0.0360)$ for corrected TBM case.

\begin{tabular}{|l||l|l|l|l|l|l|}
\hline \multicolumn{7}{|c|}{ Best Fit with latest mixing data } \\
\hline Rotation & $\chi_{\min }^{2}$ & $\theta_{12}^{\circ}$ & $\theta_{23}^{\circ}$ & $\theta_{13}^{\circ}$ & $\left|\delta_{C P}^{\circ}\right|$ & $\left|J_{C P}\right|$ \\
\hline BM & $234.7(237.5)$ & $\mathbf{4 5 . 6 ( 4 5 . 6 )}$ & $48.0(48.2)$ & $8.32(8.42)$ & $69.6(68.2)$ & $0.033(0.033)$ \\
\hline DC & $234.7(237.5)$ & $\mathbf{4 5 . 6 ( 4 5 . 6 )}$ & $48.0(48.2)$ & $8.33(8.43)$ & $36.5(39.6)$ & $0.021(0.022)$ \\
\hline TBM & $7.35(7.82)$ & $35.7(35.7)$ & $47.8(48.2)$ & $8.39(8.49)$ & $61.6(58.0)$ & $0.029(0.028)$ \\
\hline HG & $12.9(12.3)$ & $\mathbf{3 0 . 3 ( 3 0 . 3 )}$ & $47.9(48.2)$ & $8.41(8.50)$ & $52.9(49.4)$ & $0.024(0.023)$ \\
\hline
\end{tabular}

Table 5: Neutrino Mixing angles, $\left|\delta_{C P}^{\circ}\right|$ and $\left|J_{C P}\right|$ corresponding to $\chi_{\min }^{2}$ numerical fit. The mixing angle value that lies outside its best fit $3 \sigma$ range is marked in boldface.

\subsection{Rotation}

This case pertains to rotation in 23 sector of these special matrices. The expressions for neutrino in this mixing scheme are given as

$$
\begin{aligned}
\sin ^{2} \theta_{13} & =a_{12}^{2} \sin ^{2} \beta \\
\sin ^{2} \theta_{12} & =\frac{a_{12}^{2} \cos ^{2} \beta}{\cos ^{2} \theta_{13}}, \\
\sin ^{2} \theta_{23} & =\frac{a_{23}^{2} \cos ^{2} \beta+a_{22}^{2} \sin ^{2} \beta+a_{22} a_{23} \sin 2 \beta \cos \sigma}{\cos ^{2} \theta_{13}}
\end{aligned}
$$

The Jarsklog invariant and CP Dirac Phase is given by expressions 

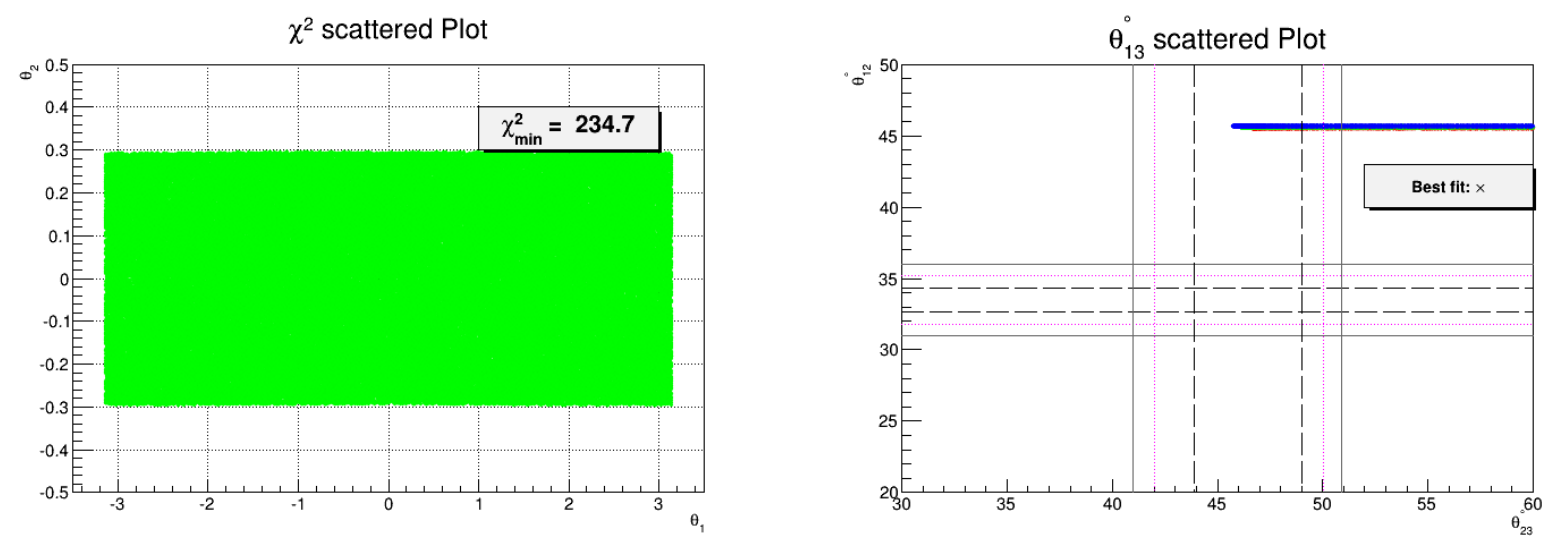

Figure 16: Scattered plot of $\chi^{2}$ (left fig.) over $\gamma-\sigma$ plane and $\theta_{13}$ (right fig.) over $\theta_{23}-\theta_{12}$ (in degrees) plane for $U_{13}^{D C R}$ rotation scheme.
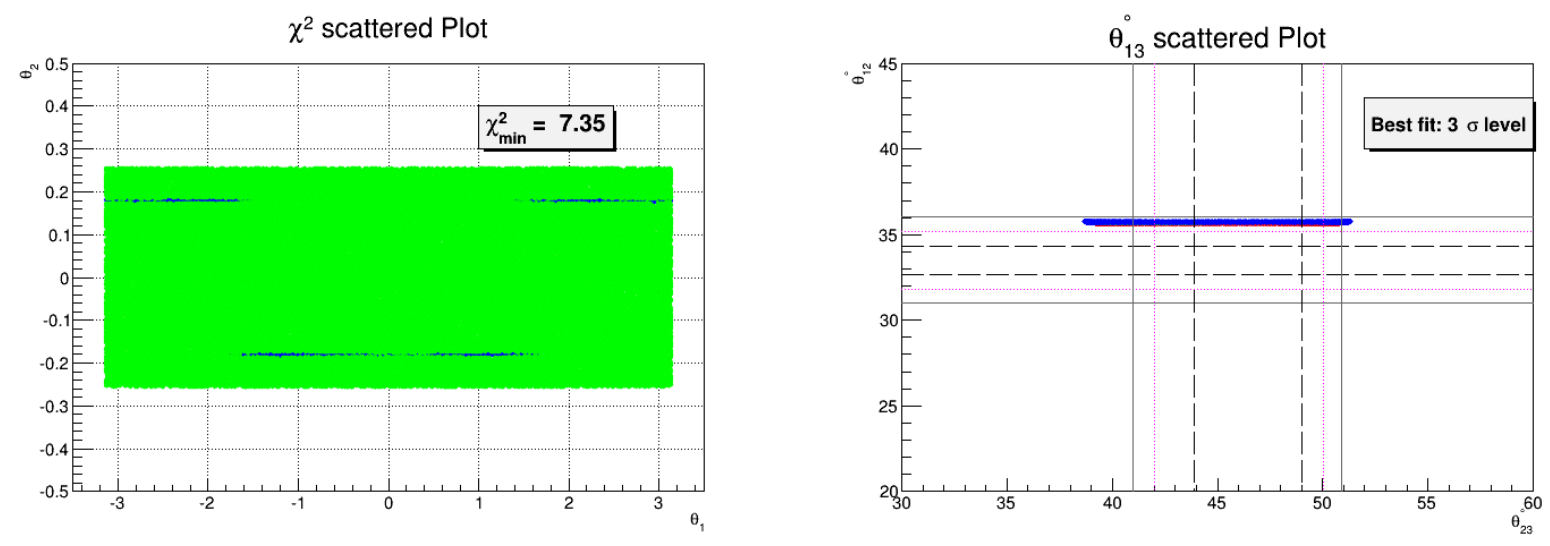

Figure 17: Scattered plot of $\chi^{2}$ (left fig.) over $\gamma-\sigma$ plane and $\theta_{13}$ (right fig.) over $\theta_{23}-\theta_{12}$ (in degrees) plane for $U_{13}^{T B M R}$ rotation scheme.

$$
\begin{aligned}
J_{C P} & =J_{23 R} \sin 2 \beta \sin \sigma \\
\sin ^{2} \delta_{C P} & =C_{23 R}^{2}\left(\frac{p_{1 \beta}}{p_{2 \beta \sigma} p_{3 \beta \sigma}}\right) \sin ^{2} \sigma
\end{aligned}
$$

where

$$
\begin{aligned}
J_{23 R} & =\frac{1}{2} a_{12}^{2} \sqrt{1-a_{12}^{2}} C_{23 R}, \\
C_{23 R} & =-\frac{a_{11} a_{21} a_{23}}{a_{12} \sqrt{1-a_{12}^{2}}}, \\
p_{1 \beta} & =1+a_{12}^{4} \sin ^{4} \beta-2 a_{12}^{2} \sin ^{2} \beta,
\end{aligned}
$$



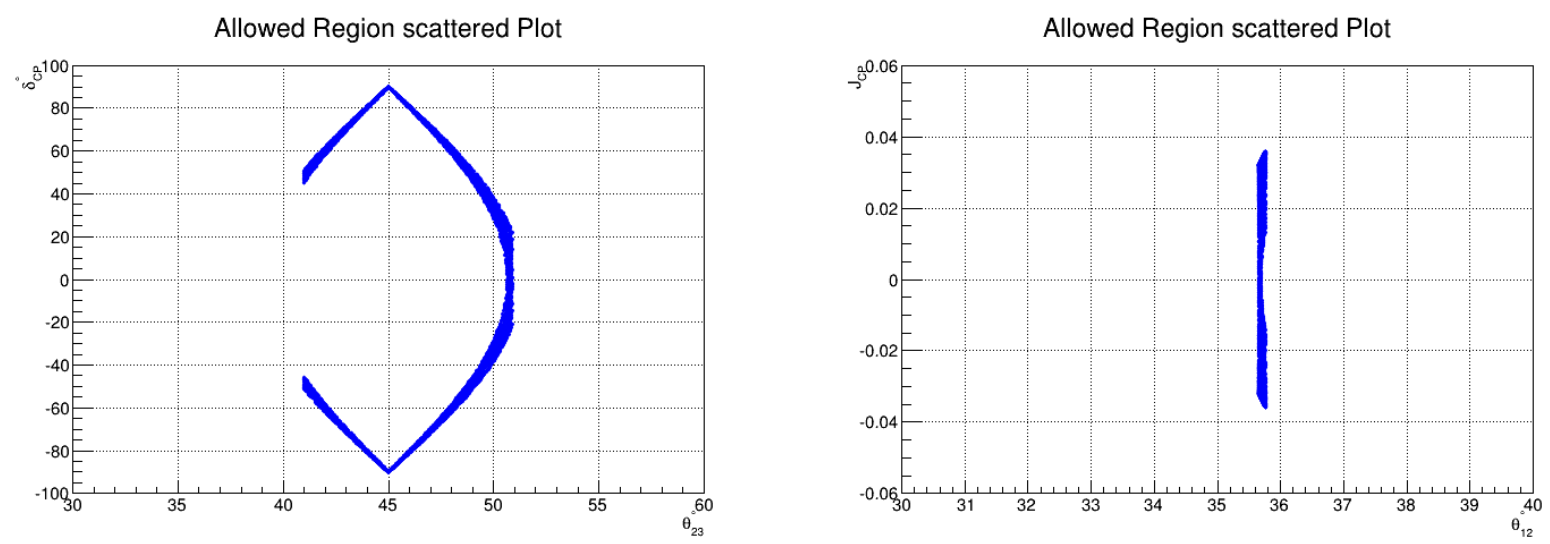

Figure 18: Scattered plot of $\delta_{C P}$ (left fig.) vs $\theta_{23}$ (in degrees) and scattered plot of $J_{C P}$ (right fig.) over $\theta_{12}$ (in degrees) plane for $U_{13}^{T B M R}$ rotation scheme.
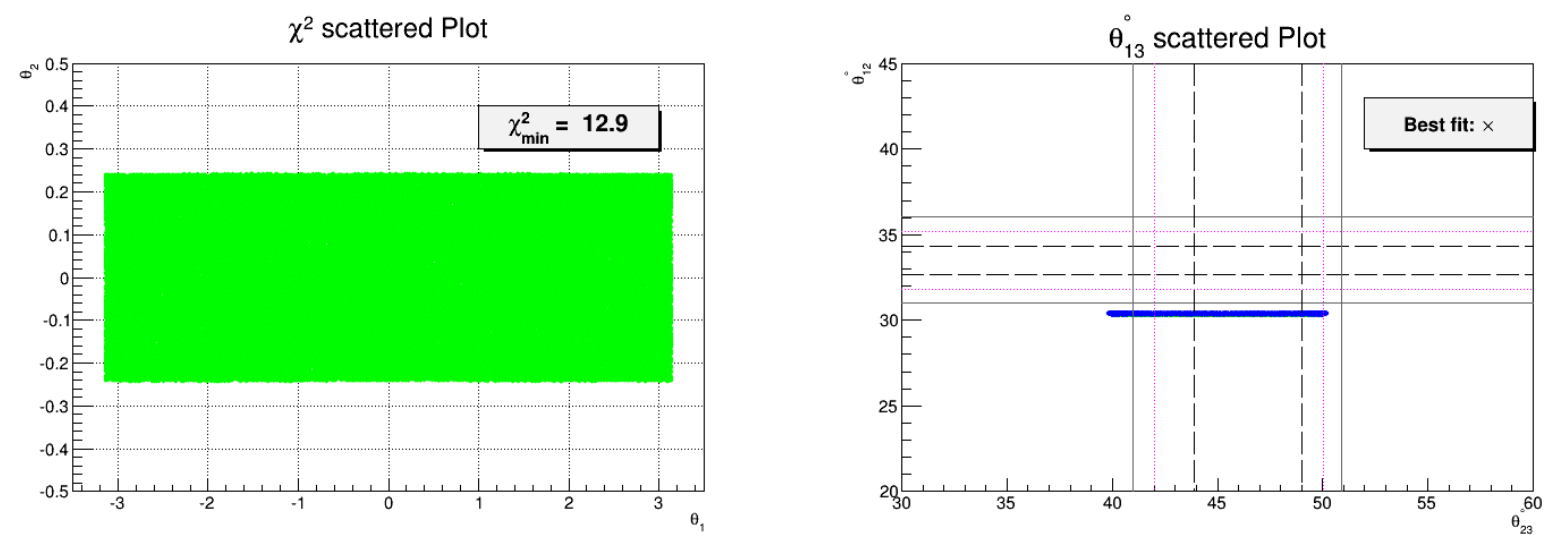

Figure 19: Scattered plot of $\chi^{2}$ (left fig.) over $\gamma-\sigma$ plane and $\theta_{13}$ (right fig.) over $\theta_{23}-\theta_{12}$ (in degrees) plane for $U_{13}^{H G R}$ rotation scheme.

$$
\begin{aligned}
& p_{2 \beta \sigma}=1-a_{23}^{2} \cos ^{2} \beta-\left(a_{12}^{2}+a_{22}^{2}\right) \sin ^{2} \beta-a_{22} a_{23} \cos \sigma \sin 2 \beta \\
& p_{3 \beta \sigma}=a_{23}^{2} \cos ^{2} \beta+a_{22}^{2} \sin ^{2} \beta+a_{22} a_{23} \cos \sigma \sin 2 \beta
\end{aligned}
$$

Fig. 20-23 show the numerical results corresponding to this mixing scheme. The salient features in this perturbative scheme are:

(i) Like previous case, $\theta_{12}$ receives corrections only of the $O\left(\theta^{2}\right)$ so its value remains close to its original prediction. Thus BM and DC will not be preferred in this mixing scheme.

(ii) As fitting of $\theta_{13}$ and $\theta_{12}$ is only governed by $\beta$ so its allowed range is much constrained in parameter space. e.g. for TBM case, the fitting of $\theta_{13}$ under its $3 \sigma$ domain constraints the magnitude of correction parameter $|\alpha| \in[0.2412(0.2443), 0.2711(0.2734)]$ which in turn fixes $\theta_{12} \in\left[34.26^{\circ}\left(34.24^{\circ}\right), 34.47^{\circ}\left(34.45^{\circ}\right)\right]$ for corresponding $\beta$ values with $\mathrm{NH}(\mathrm{IH})$. However $\theta_{23}$ possess much wider range of values since it receives corrections from $\beta$ as well from phase 

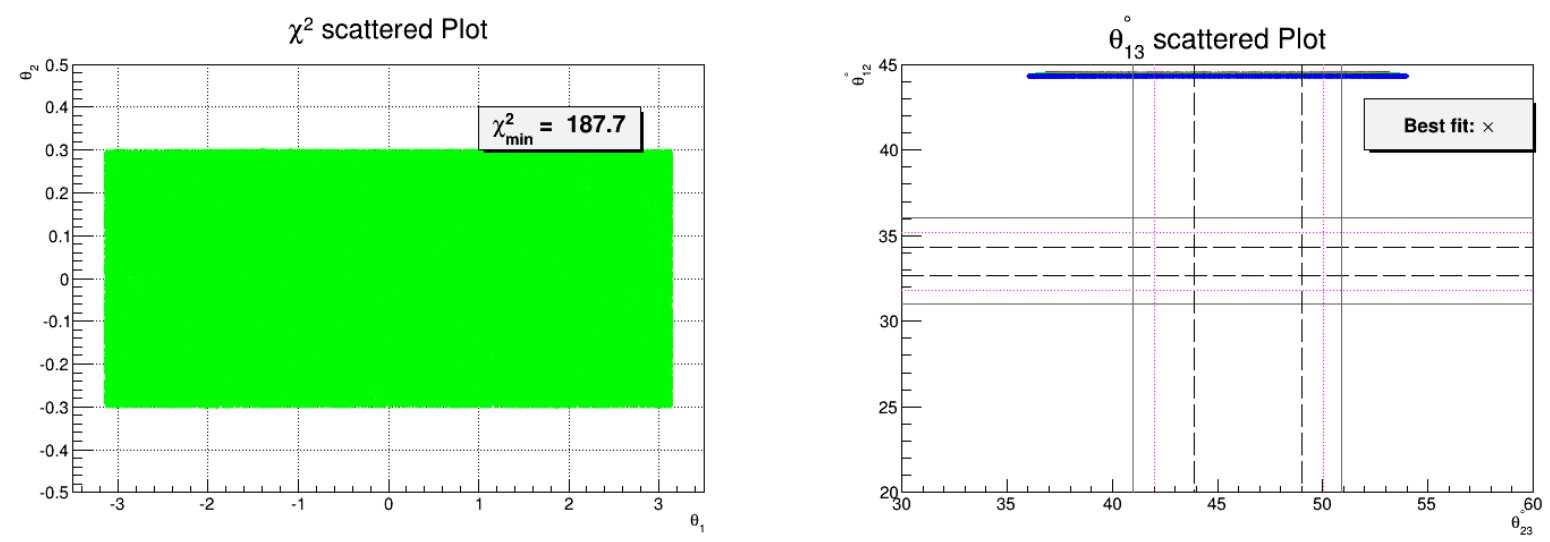

Figure 20: Scattered plot of $\chi^{2}$ (left fig.) over $\beta-\sigma$ plane and $\theta_{13}$ (right fig.) over $\theta_{23}-\theta_{12}$ (in degrees) plane for $U_{23}^{B M R}$ rotation scheme.

parameter $\sigma$.

(iii) The minimum value of $\chi^{2} \sim 187.7(189.3), 187.7(189.3), 1.19(1.31)$ and $40.8(86.7)$ for BM, DC, TBM and HG respectively with $\mathrm{NH}(\mathrm{IH})$ case. Here BM, DC and HG are unable to bring $\theta_{12}$ in its allowed range so these cases are not consistent. However TBM is much favored as it can fit all mixing angles within $1 \sigma$ range for $\mathrm{NH}$ and $\mathrm{IH}$.

(iv) Leptonic phase $\delta_{C P}$ lies in the range 60.1(60.3) $\leq\left|\delta_{C P}\right| \leq 89.9(89.9)$ and $J_{C P}$ confined in range $0.026(0.027) \leq\left|J_{C P}\right| \leq 0.035(0.035)$ for TBM matrix.

\begin{tabular}{|l||l|l|l|l|l|l|}
\hline \multicolumn{7}{|c|}{ Best Fit with Mixing data } \\
\hline Rotation & $\chi_{\min }^{2}$ & $\theta_{12}^{\circ}$ & $\theta_{23}^{\circ}$ & $\theta_{13}^{\circ}$ & $\left|\delta_{C P}^{\circ}\right|$ & $\left|J_{C P}\right|$ \\
\hline BM & $187.7(189.3)$ & $\mathbf{4 4 . 3 ( 4 4 . 3 )}$ & $47.9(48.3)$ & $8.48(8.54)$ & $70.2(67.9)$ & $0.033(0.033)$ \\
\hline DC & $187.7(189.3)$ & $\mathbf{4 4 . 3 ( 4 4 . 3 )}$ & $47.7(48.1)$ & $8.47(8.54)$ & $34.7(39.4)$ & $0.020(0.022)$ \\
\hline TBM & $1.19(1.31)$ & $34.3(34.3)$ & $47.8(48.2)$ & $8.41(8.50)$ & $77.0(75.2)$ & $0.032(0.032)$ \\
\hline HG & $40.8(86.7)$ & $\mathbf{2 8 . 9}(\mathbf{2 8 . 9})$ & $\mathbf{5 7 . 7}(\mathbf{5 7 . 6})$ & $8.33(8.23)$ & $32.0(31.9)$ & $0.014(0.014)$ \\
\hline
\end{tabular}

Table 6: Neutrino Mixing angles, $\left|\delta_{C P}^{\circ}\right|$ and $\left|J_{C P}\right|$ corresponding to $\chi_{\min }^{2}$ numerical fit. The mixing angle value that lies outside its best fit $3 \sigma$ range is marked in boldface.

\section{Summary and Conclusions}

Tribimaximal(TBM), Bimaximal(BM), Democratic(DC) and Hexagonal(HG) mixing attracted much attention in literature for explaining the neutrino mixing data. All these scenarios comes with a common prediction of vanishing reactor mixing angle. The atmospheric mixing angle $\left(\theta_{23}\right)$ is maximal in TBM, BM and $\mathrm{HG}$ mixing while it takes a larger 

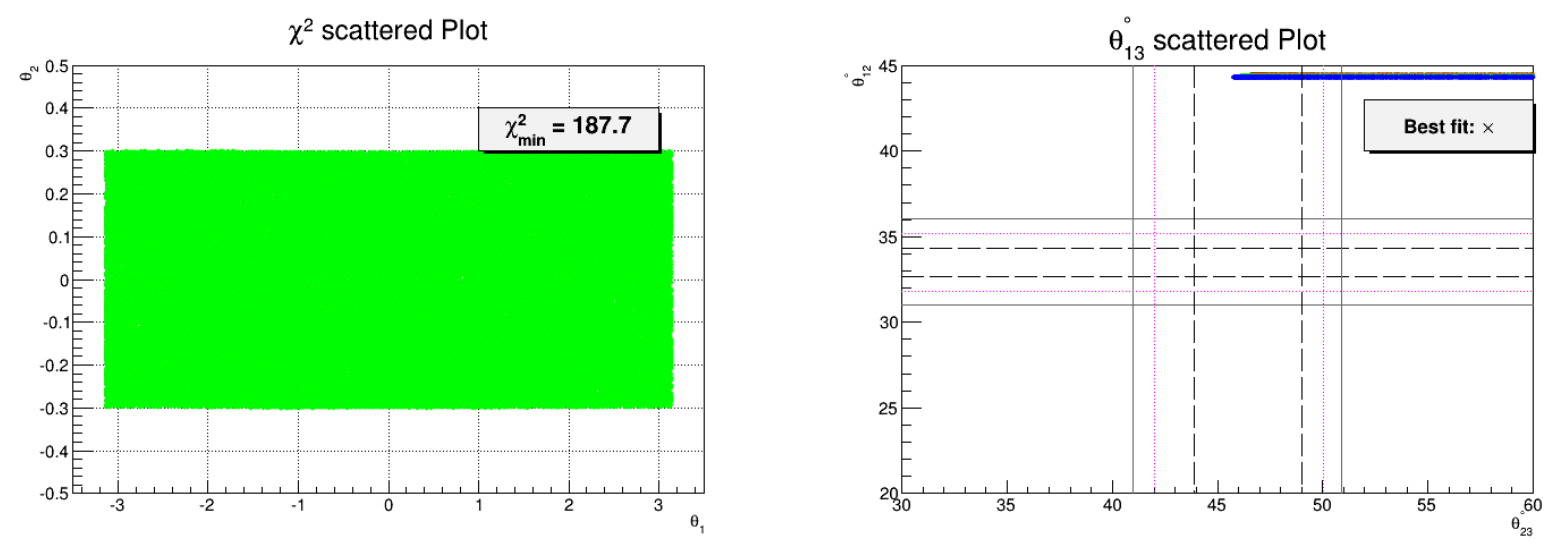

Figure 21: Scattered plot of $\chi^{2}$ (left fig.) over $\beta-\sigma$ plane and $\theta_{13}$ (right fig.) over $\theta_{23}-\theta_{12}$ (in degrees) plane for $U_{23}^{D C R}$ rotation scheme.
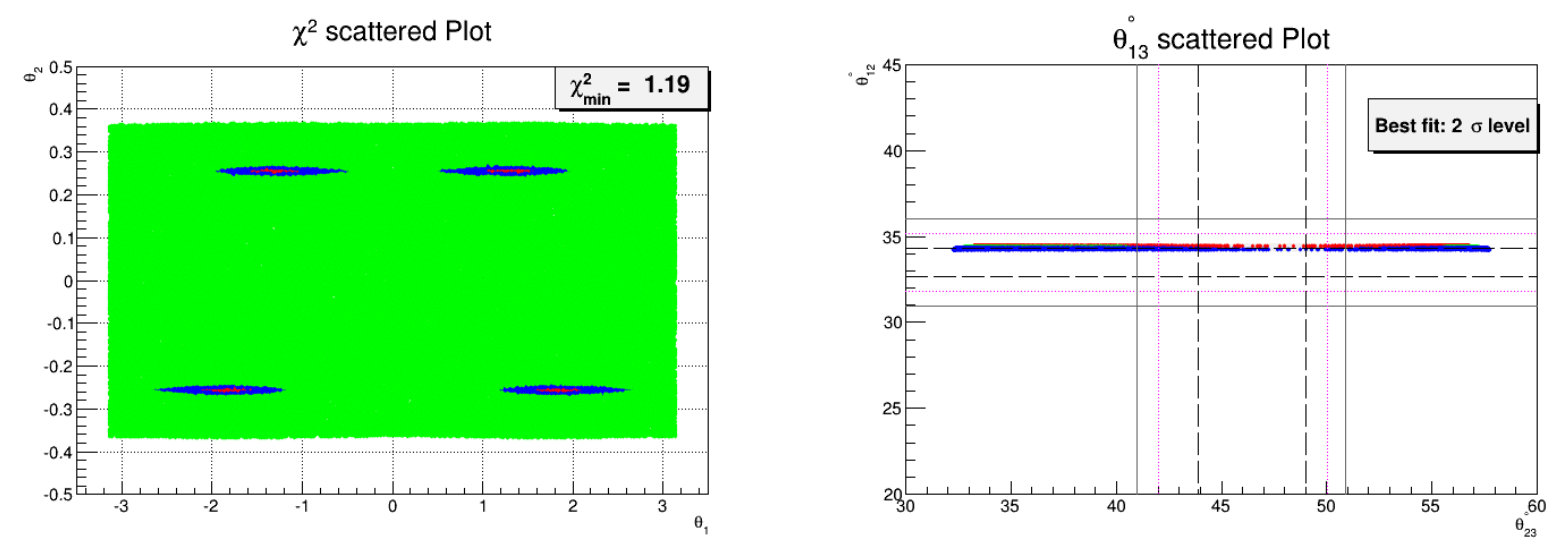

Figure 22: Scattered plot of $\chi^{2}$ (left fig.) over $\beta-\sigma$ plane and $\theta_{13}$ (right fig.) over $\theta_{23}-\theta_{12}$ (in degrees) plane for $U_{23}^{T B M R}$ rotation scheme.

value of $54.7^{\circ}$ for DC case. The solar mixing angle $\left(\theta_{12}\right)$ is maximal in BM and DC scenarios while its value is $35.3^{\circ}$ and $30.0^{\circ}$ for TBM and $\mathrm{HG}$ case respectively. However experimental observation of non zero reactor mixing angle $\left(\theta_{13} \approx 8^{\circ}\right)$ and departure of other two mixing angles from maximality is asking for corrections in these mixing schemes.

In this study, we performed a detailed analysis of corrections around these mixing scenarios. These modifications are expressed in terms of three Unitary rotation matrices $\mathrm{U}_{12}, \mathrm{U}_{13}$ and $\mathrm{U}_{23}$ which acts on 12, 13 and 23 sector of unperturbed PMNS matrix respectively. We investigated all possible cases that are governed by one rotation matrix with corresponding modified PMNS matrices of the forms $\left(U_{i j} \cdot V_{M}, V_{M} \cdot U_{i j}\right)$ where $V_{M}$ is any one of these special matrices. Here $U_{i j}$ is a complex rotation matrix that will act on $i j$ sector of unperturbed mixing matrix and is described by a rotation angle and a phase parameter. As the form of PMNS matrix is given by $U_{P M N S}=U_{l}^{\dagger} U_{\nu}$ so these corrections can originate from charged 

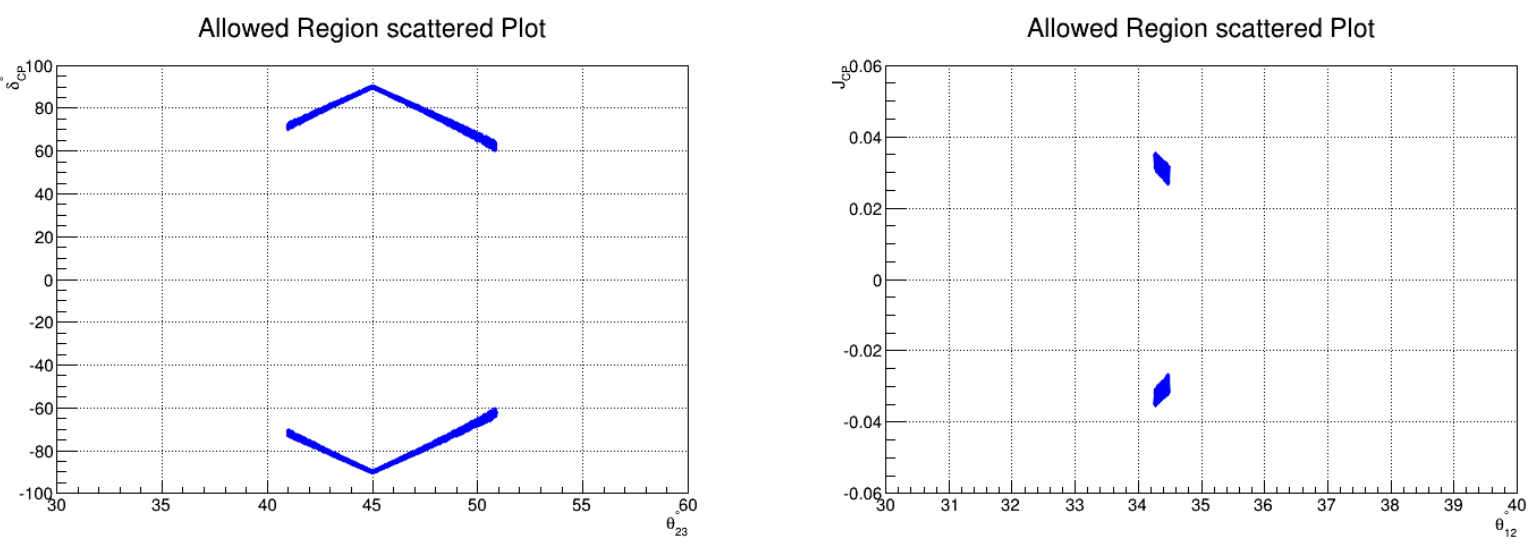

Figure 23: Scattered plot of $\delta_{C P}$ (left fig.) vs $\theta_{23}$ (in degrees) and scattered plot of $J_{C P}$ (right fig.) over $\theta_{12}$ (in degrees) plane for $U_{23}^{T B M R}$ rotation scheme.
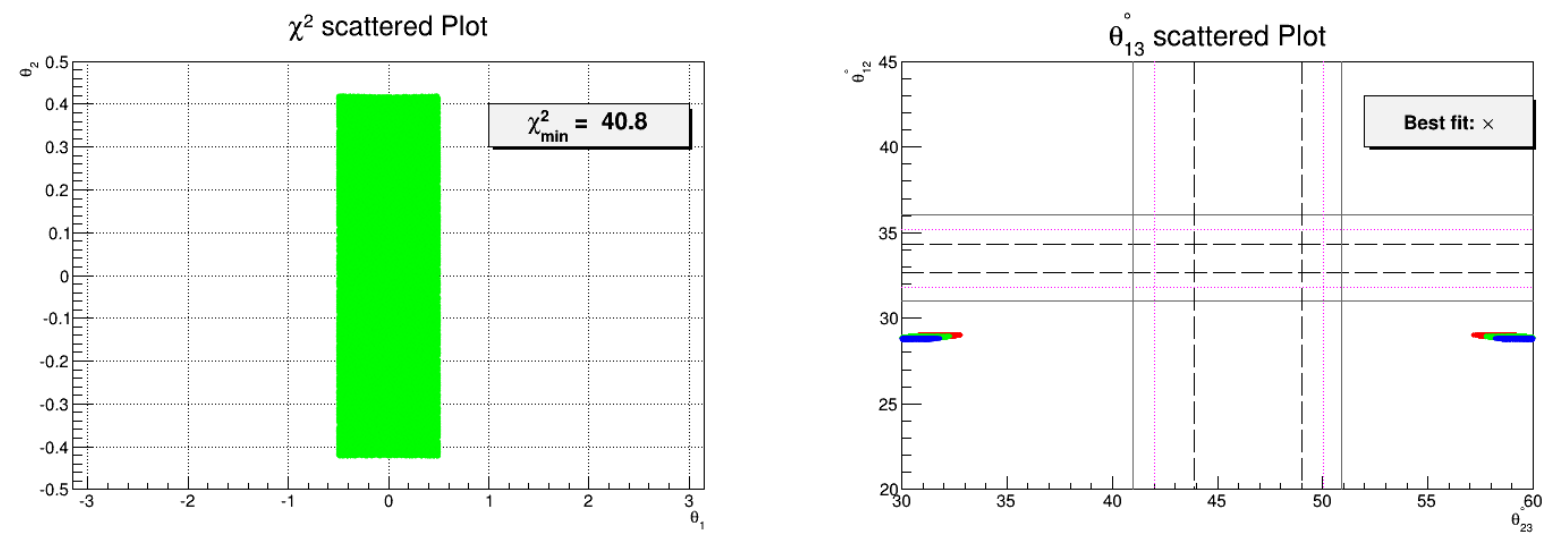

Figure 24: Scattered plot of $\chi^{2}$ (left fig.) over $\beta-\sigma$ plane and $\theta_{13}$ (right fig.) over $\theta_{23}-\theta_{12}$ (in degrees) plane for $U_{23}^{H G R}$ rotation scheme.

lepton and neutrino sector respectively. For our numerical analysis, we invoked $\chi^{2}$ function which is a combined measure of deviation of values of mixing angles in parameter space to that coming from experimental best fit values. The resulting value of $\delta_{C P}$ and $J_{C P}$ in allowed parameter space is treated as the prediction of that particular mixing scheme. The numerical findings are presented in terms of $\chi^{2}$ vs perturbation parameters and as correlations among different neutrino mixing angles. The scattered plots for $\delta_{C P}$ and $J_{C P}$ vs mixing angles are also presented for determining the allowed ranges of these quantities. In Table 7 and Table 8 we presented the formulae for mixing angles, $\delta_{C P}$ and $J_{C P}$ for all considered cases. Table 9 contains final results of our investigation in terms of $\left(\chi^{2}\right.$, Best Fit). The obtained ranges of $\delta_{C P}$ and $J_{C P}$ are given in Table 9 and Table 10 respectively.

The mixing $U_{12} \cdot V_{M}$ imparts $O\left(\theta^{2}\right)$ corrections to $\theta_{23}$ and thus it prefers to stay close to its unperturbed prediction in parameter space. However $\theta_{12}$ can possess wide range of 
values since it gets correction from rotation as well as phase parameter. Since for DC case $\theta_{23} \sim 54.7^{\circ}$ so it is disfavored completely. The modified BM mixing can fit all mixing angles within $3 \sigma$ range with $\chi_{\min }^{2} \sim 20.2(30.2)$ whereas TBM and HG can fit angles in $1 \sigma(2 \sigma)$ range with $\chi_{\text {min }}^{2} \sim 1.95(11.0)$ and $\chi_{\text {min }}^{2} \sim 1.94(11.0)$ respectively for Normal Hierarchy(Inverted Hierarchy). Thus this mixing scheme shows preference towards NH in parameter space. As far as leptonic CP phase is concerned, it prefers a smaller value of $\left[-4.4^{\circ}\left(-4.7^{\circ}\right) \leq\right.$ $\left.\delta_{C P} \leq 3.4^{\circ}\left(5.3^{\circ}\right)\right]$ with corresponding $\left[-0.0027(-0.0029) \leq J_{C P} \leq 0.0021(0.0033)\right]$ in allowed parameter space for modified BM case. However allowed parameter space prefers larger value of $\mathrm{CP}$ phase in range $\left[61.0^{\circ}\left(60.9^{\circ}\right) \leq\left|\delta_{C P}\right| \leq 89.9^{\circ}\left(89.9^{\circ}\right)\right]$ and $\left[39.0^{\circ}\left(40.4^{\circ}\right) \leq\right.$ $\left.\left|\delta_{C P}\right| \leq 78.7^{\circ}\left(79.2^{\circ}\right)\right]$ with corresponding $J_{C P}$ in the range $\left[0.026 \leq\left|J_{C P}\right| \leq 0.035\right]$ and $\left[0.020(0.021) \leq\left|J_{C P}\right| \leq 0.032(0.032)\right]$ for corrected TBM and BM respectively.

The $U_{13} \cdot V_{M}$ mixing scheme is quite similar to previous case. Here $\theta_{23}$ receives very minor corrections which enters through $\theta_{13}$. Thus modified value of $\theta_{23}$ remains quite close to its original prediction. However $\theta_{12}$ can have wide range in parameter space since it receives correction from rotation as well as phase parameter. Here also DC case is not viable. The modified BM can fit all mixing angles within $3 \sigma$ range with $\chi_{\min }^{2} \sim 18.9(23.4)$ while TBM and HG can fit all angles in $1 \sigma(2 \sigma)$ range with $\chi_{\min }^{2} \sim 0.82(5.0)$ and $\chi_{\min }^{2} \sim 0.81(5.0)$ respectively. Thus this case also prefers $\mathrm{NH}$ and overall picture of fitting is better then previous scheme due to lower value of $\chi_{\text {min }}^{2}$. The leptonic phase $\delta_{C P}$ prefers a smaller value of $\left[-3.6^{\circ}\left(-4.9^{\circ}\right) \leq\right.$ $\left.\delta_{C P} \leq 4.2^{\circ}\left(5.5^{\circ}\right)\right]$ with corresponding $\left[-0.0025(-0.0034) \leq J_{C P} \leq 0.0026(0.0030)\right]$ in allowed parameter space for modified BM case. However allowed parameter have same ranges of $\delta_{C P}$ and $J_{C P}$ as that obtained in previous case for $U_{12} \cdot V_{M}$ mixing.

For $U_{23} \cdot V_{M}$ and $V_{M} \cdot U_{12}$ mixing scheme, 13 element of modified matrix is still zero and thus $\theta_{13}$ remains stick to its unperturbed value i.e. $\theta_{13}=0$. Thus these two cases are not suitable for any further investigation.

For $V_{M} \cdot U_{13}$ mixing scheme, $\theta_{12}$ receives very minor corrections only through $\theta_{13}$ and thus it remains quite close to its unperturbed value. However $\theta_{23}$ can possess wide range of values in parameter space since it receives correction from rotation as well as phase parameter. Thus BM, DC and HG are not favorable as their unperturbed values are outside $3 \sigma$ range. However TBM is still consistent as its unperturbed value is $\theta_{12} \sim 35.3^{\circ}$ which lies within global fit $3 \sigma$ range. It can fit all mixing angles with $\chi_{\min }^{2} \sim 7.35(7.82)$ for $\mathrm{NH}(\mathrm{IH})$. This mixing scheme allows much larger range of $\left[-89.9^{\circ} \leq \delta_{C P} \leq 89.9^{\circ}\right]$ and $\left[-0.035(-0.036) \leq J_{C P} \leq 0.035(0.036)\right]$ in allowed parameter space for $\mathrm{NH}$ and $\mathrm{IH}$. Thus overall this mixing scheme is not much favorable.

In this case, $\theta_{12}$ receives $O\left(\theta^{2}\right)$ corrections and thus its modified value remains close to its unperturbed value. However $\theta_{23}$ can have wide range of values in parameter space as it gets corrections from rotation and phase parameter. The BM, DC and HG are not viable 
but TBM is preferable as it can fit all mixing angles within $2 \sigma$ level with $\chi_{\min }^{2} \sim 1.19(1.31)$. The predicted value of Leptonic $\mathrm{CP}$ phase lies in range $\left[60.1^{\circ}\left(60.3^{\circ}\right) \leq\left|\delta_{C P}\right| \leq 89.9^{\circ}\left(89.9^{\circ}\right)\right]$ for $\mathrm{NH}(\mathrm{IH})$. However $J_{C P}$ remains in range $\left[0.026(0.027) \leq\left|J_{C P}\right| \leq 0.035(0.035)\right]$ for $\mathrm{NH}$ as well as IH.

This completes our discussion on checking the consistency of these schemes with mixing data and corresponding prediction for CP Violating Phase and Jarkslog invariant for various cases. This model independent study might turn out to be useful in restricting vast number of possible models which offers different corrections to this mixing scheme in neutrino model building physics. It thus can be a guideline for neutrino model building. Moreover the predictions of these mixing scenarios can be checked from current and future neutrino experiments.

\section{Acknowledgments}

The author is thankful to Prof. B. Ananthnarayan for hospitality during visit to CHEP, IISC Bengaluru where version v2 of this study was completed.

\section{A Results: Summary}

In this appendix, we collect all our formulae and results for considered mixing schemes. In Table 7 and Table 8, we supplied the expressions for mixing angles, Dirac CP Phase $\left(\delta_{C P}\right)$ and Jarkslog invariant $\left(J_{C P}\right)$ in terms of correction parameters. In Table 9 , we presented $\left(\chi_{\min }^{2}\right.$, Best Fit) for various studied cases while in Table 10 and Table 11 we gave allowed ranges for $\delta_{C P}$ and $J_{C P}$ respectively. 


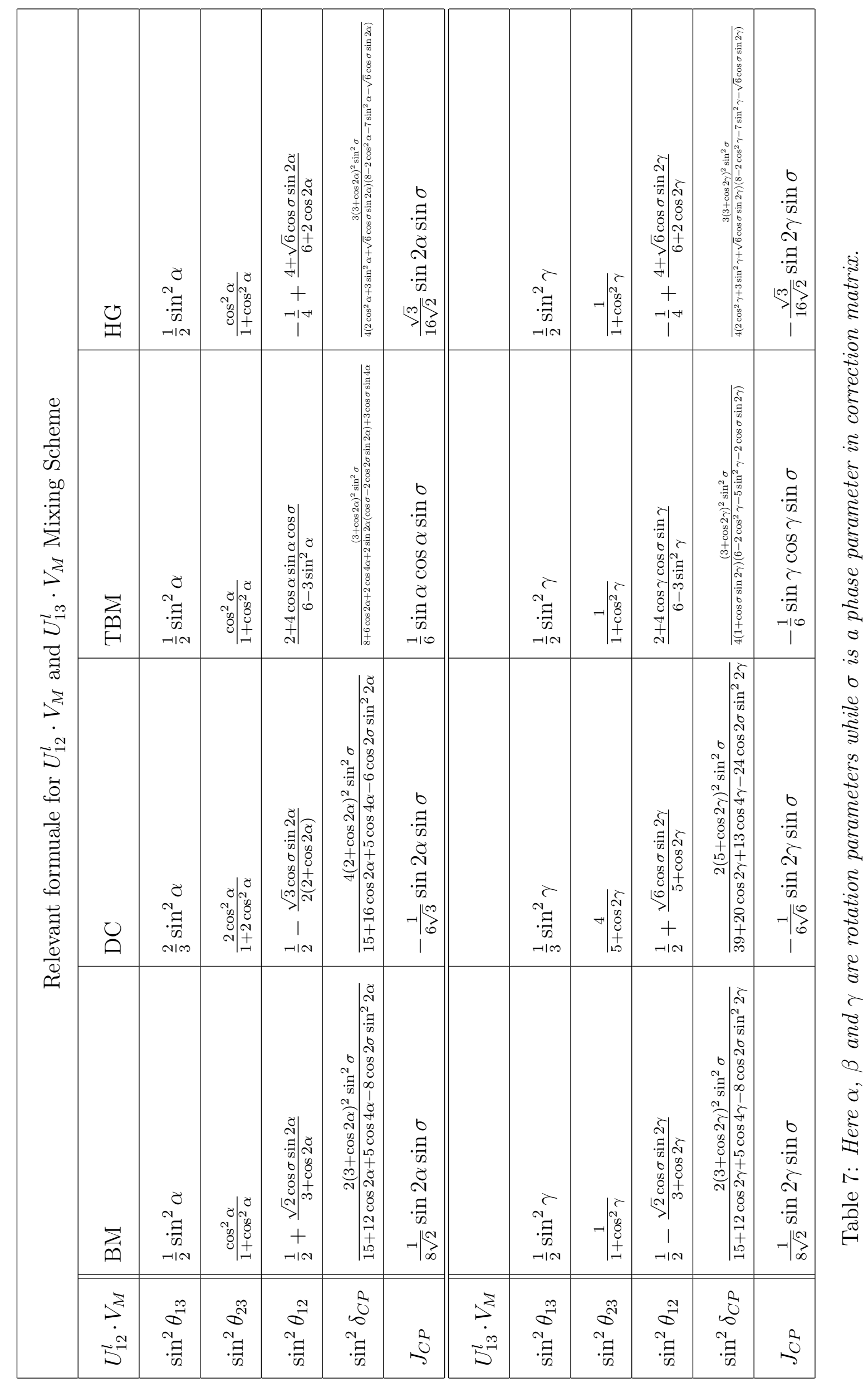




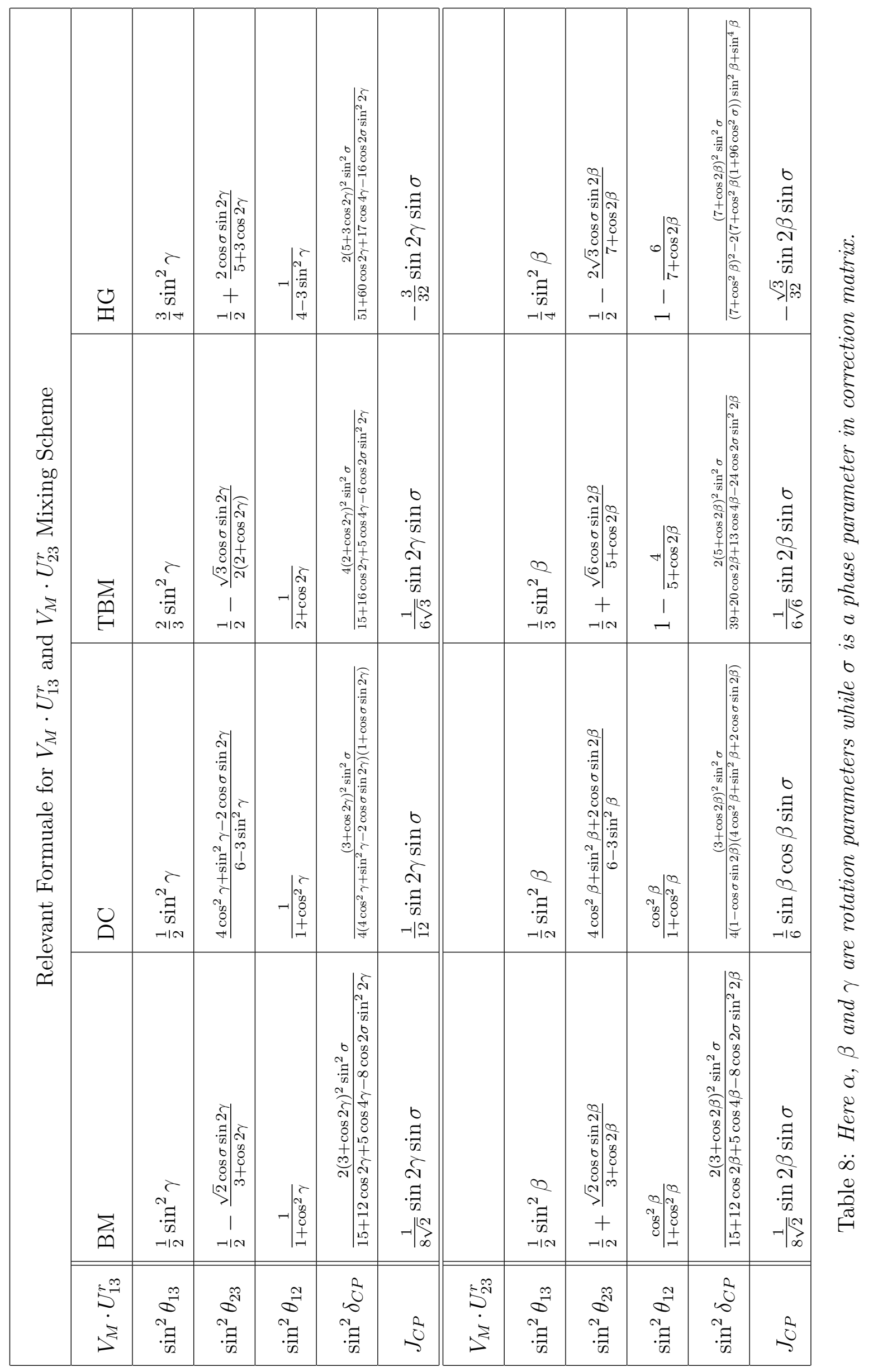




\begin{tabular}{|l||l|l|l|l|}
\hline \multicolumn{5}{|c|}{$\left(\chi_{\min }^{2}\right.$, Best fit level) for NH and IH from Mixing angles fitting } \\
\hline $\begin{array}{l}\text { Rotation- } \\
\text { NH }\end{array}$ & BM & DC & TBM & HG \\
\hline$U_{12}^{l}$ & $(20.2,3 \sigma)$ & $(51.4, \times)$ & $(1.95,1 \sigma)$ & $(1.94,1 \sigma)$ \\
\hline$U_{13}^{l}$ & $(18.9,3 \sigma)$ & $(8.56, \times)$ & $(0.82,1 \sigma)$ & $(0.81,1 \sigma)$ \\
\hline$U_{13}^{r}$ & $(234.7, \times)$ & $(234.7, \times)$ & $(7.35,3 \sigma)$ & $(12.9, \times)$ \\
\hline$U_{23}^{r}$ & $(187.7, \times)$ & $(187.7, \times)$ & $(1.19,2 \sigma)$ & $(40.8, \times)$ \\
\hline \hline Rotation-IH & & & & \\
\hline$U_{12}^{l}$ & $(30.2,3 \sigma)$ & $(70.4, \times)$ & $(11.0,2 \sigma)$ & $(11.0,2 \sigma)$ \\
\hline$U_{13}^{l}$ & $(23.4,3 \sigma)$ & $(36.8, \times)$ & $(5.0,2 \sigma)$ & $(5.0,2 \sigma)$ \\
\hline$U_{13}^{r}$ & $(237.5, \times)$ & $(237.5, \times)$ & $(7.82,3 \sigma)$ & $(12.3, \times)$ \\
\hline$U_{23}^{r}$ & $(189.3, \times)$ & $(189.3, \times)$ & $(1.31,2 \sigma)$ & $(86.7, \times)$ \\
\hline
\end{tabular}

Table 9: Here ' $\times$ ' refers to the case which is unable to fit mixing angles even at $3 \sigma$ level. 

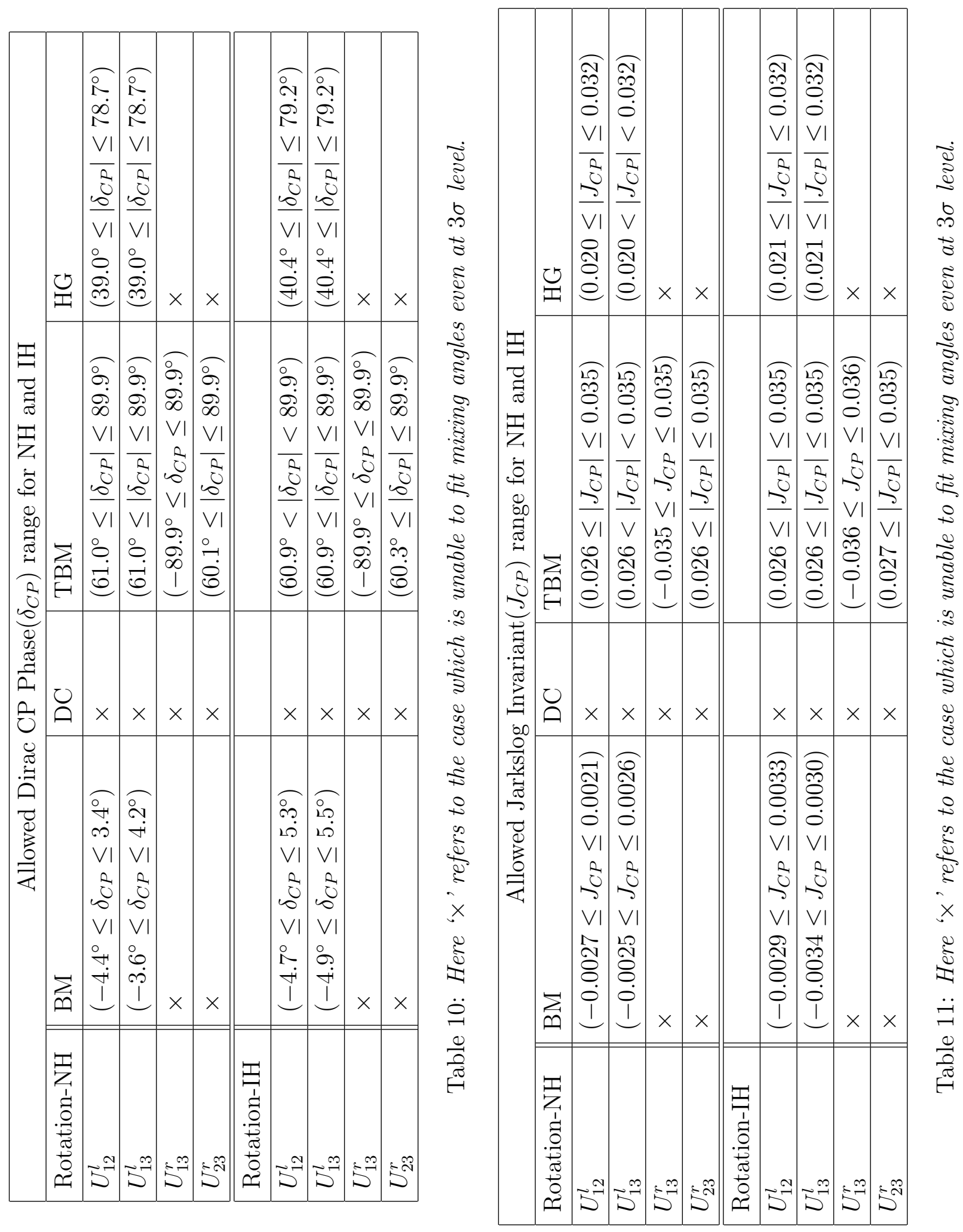


\section{References}

[1] Y. Fukuda et al. [Super-Kamiokande Collaboration], Phys. Rev. Lett. 81, 1562 (1998) [hep-ex/9807003].

[2] A. B. McDonald, Rev. Mod. Phys. 88, no. 3, 030502 (2016).

[3] T. Kajita, Rev. Mod. Phys. 88, no. 3, 030501 (2016).

[4] F. P. An et al. [DAYA-BAY Collaboration], Observation of electron-antineutrino disappearance at Daya Bay, Phys. Rev. Lett. 108, 171803 (2012) [arXiv:1203.1669 [hep-ex]].

[5] K. Abe et al. [T2K Collaboration], Phys. Rev. Lett. 107, 041801 (2011) [arXiv:1106.2822 [hep-ex]].

[6] Y. Abe et al. [DOUBLE-CHOOZ Collaboration], Indication for the disappearance of reactor electron antineutrinos in the Double Chooz experiment, Phys. Rev. Lett. 108, 131801 (2012) [arXiv:1112.6353 [hep-ex]].

[7] P. Adamson et al. [MINOS Collaboration], Improved search for muon-neutrino to electron-neutrino oscillations in MINOS, Phys. Rev. Lett. 107, 181802 (2011) [arXiv:1108.0015 [hep-ex]].

[8] J. K. Ahn et al. [RENO Collaboration], Observation of Reactor Electron Antineutrino Disappearance in the RENO Experiment, Phys. Rev. Lett. 108, 191802 (2012) [arXiv:1204.0626 [hep-ex]].

[9] B. Pontecorvo, Zh. Eksp. Teor. Fiz. (JTEP) 33, 549 (1957); ibid. 34, 247 (1958); ibid. 53, 1717 (1967); Z. Maki, M. Nakagawa and S. Sakata, Prog. Theor. Phys. 28, 870 (1962).

[10] I. Esteban, M. C. Gonzalez-Garcia, M. Maltoni, I. Martinez-Soler and T. Schwetz, JHEP 1701, 087 (2017) [arXiv:1611.01514 [hep-ph]].

[11] F. Capozzi, E. Di Valentino, E. Lisi, A. Marrone, A. Melchiorri and A. Palazzo, Phys. Rev. D 95, no. 9, 096014 (2017) [arXiv:1703.04471 [hep-ph]].

[12] P. F. de Salas, D. V. Forero, C. A. Ternes, M. Tortola and J. W. F. Valle, arXiv:1708.01186 [hep-ph].

[13] NuFIT webpage http://www.nu-fit.org (v3.2: Three-neutrino flavor fit based on data available in January 2018).

[14] F. Capozzi, E. Lisi, A. Marrone and A. Palazzo, arXiv:1804.09678 [hep-ph]. 
[15] K. Abe et al. [T2K Collaboration], Phys. Rev. Lett. 112, 061802 (2014) [arXiv:1311.4750 [hep-ex]].

[16] P. Adamson et al. [NOvA Collaboration], Phys. Rev. Lett. 118, no. 23, 231801 (2017) [arXiv:1703.03328 [hep-ex]].

[17] I. Stancu, D. V. Ahluwalia and , L / E flatness of the electron - like event ratio in Super-Kamiokande and a degeneracy in neutrino masses, Phys. Lett. B 460, 431 (1999) [hep-ph/9903408].

[18] P. F. Harrison, D. H. Perkins, W. G. Scott, Tri-Bimaximal Mixing and the Neutrino Oscillation Data, Phys. Lett. B 530, 167 (2002) [arXiv:0202074 [hep-ph]].

[19] P. F. Harrison, W. G. Scott, Symmetries and Generalisations of Tri-Bimaximal Neutrino Mixing, Phys. Lett. B 535, 163 (2002) [arXiv:0203209 [hep-ph]].

[20] Zhi zhong Xing, Nearly Tri-Bimaximal Neutrino Mixing and CP Violation, Phys. Lett. B 533, 85 (2002) [arXiv:0204049 [hep-ph]].

[21] G. Altarelli, F. Feruglio, L. Merlo and E. Stamou, Discrete Flavour Groups, $\theta_{13}$ and Lepton Flavour Violation, JHEP 1208, 021 (2012) [arXiv:1205.4670 [hep-ph]].

[22] F. Vissani, A Study of the scenario with nearly degenerate Majorana neutrinos,' hep$\mathrm{ph} / 9708483$.

[23] V. D. Barger, S. Pakvasa, T. J. Weiler, K. Whisnant, Bi-Maximal Mixing of Three Neutrinos, Phys. Lett. B 467107 (1998) [arXiv:9806387 [hep-ph]].

[24] A. J. Baltz, A. S. Goldhaber, M. Goldhaber, The Solar Neutrino Puzzle: An Oscillation Solution with Maximal Neutrino Mixing, Phys. Rev. Lett. 815730 (1998) [arXiv:9806540 [hep-ph]].

[25] D. V. Ahluwalia and, Reconciling Super-Kamiokande, LSND, and home-stake neutrino oscillation data, Mod. Phys. Lett. A 13, 2249 (1998) [hep-ph/9807267].

[26] H. J. He, D. A. Dicus and J. N. Ng, Phys. Lett. B 536, 83 (2002) [hep-ph/0203237].

[27] G. Altarelli, F. Feruglio and L. Merlo, Revisiting Bimaximal Neutrino Mixing in a Model with S(4) Discrete Symmetry, JHEP 0905, 020 (2009) [arXiv:0903.1940 [hep-ph]].

[28] H. J. He and X. J. Xu, Phys. Rev. D 86, 111301 (2012) [arXiv:1203.2908 [hep-ph]].

[29] G. Altarelli, F. Feruglio, L. Merlo and E. Stamou, Discrete Flavour Groups, theta ${ }_{13}$ and Lepton Flavour Violation, JHEP 1208, 021 (2012) [arXiv:1205.4670 [hep-ph]]. 
[30] H. Fritzsch and Z. -Z. Xing, Lepton mass hierarchy and neutrino oscillations, Phys. Lett. B 372, 265 (1996) [hep-ph/9509389].

[31] H. Fritzsch and Z. -z. Xing, Large leptonic flavor mixing and the mass spectrum of leptons, Phys. Lett. B 440, 313 (1998) [hep-ph/9808272].

[32] Z. -Z. Xing, The T2K Indication of Relatively Large $\theta_{13}$ and a Natural Perturbation to the Democratic Neutrino Mixing Pattern, Chin. Phys. C 36, 101 (2012) [arXiv:1106.3244 [hep-ph]].

[33] C. H. Albright, A. Dueck and W. Rodejohann, Possible Alternatives to Tri-bimaximal Mixing, Eur. Phys. J. C 70, 1099 (2010) [arXiv:1004.2798 [hep-ph]].

[34] S. Zhou, Relatively large theta13 and nearly maximal theta23 from the approximate S3 symmetry of lepton mass matrices, Phys. Lett. B 704, 291 (2011) [arXiv:1106.4808 [hep-ph]].

[35] T. Araki, Getting at large $\theta_{13}$ with almost maximal $\theta_{23}$ from tri-bimaximal mixing, Phys. Rev. D 84, 037301 (2011) [arXiv:1106.5211 [hep-ph]].

[36] W. Chao and Y. -j. Zheng, Relatively Large Theta13 from Modification to the Tribimaximal, Bimaximal and Democratic Neutrino Mixing Matrices, JHEP 1302, 044 (2013) [arXiv:1107.0738 [hep-ph]].

[37] H. Zhang and S. Zhou, Radiative corrections and explicit perturbations to the tetramaximal neutrino mixing with large $\theta_{13}$, Phys. Lett. B 704, 296 (2011) [arXiv:1107.1097 [hep-ph]].

[38] W. Rodejohann, H. Zhang and S. Zhou, Systematic search for successful lepton mixing patterns with nonzero $\theta_{13}$, Nucl. Phys. B 855, 592 (2012) [arXiv:1107.3970 [hep-ph]].

[39] S. Antusch, S. F. King, C. Luhn and M. Spinrath, Trimaximal mixing with predicted $\theta_{13}$ from a new type of constrained sequential dominance, Nucl. Phys. B 856, 328 (2012) [arXiv:1108.4278 [hep-ph]].

[40] S. -F. Ge, D. A. Dicus and W. W. Repko, Residual Symmetries for Neutrino Mixing with a Large $\theta_{13}$ and Nearly Maximal $\delta_{D}$, Phys. Rev. Lett. 108, 041801 (2012) [arXiv:1108.0964 [hep-ph]].

[41] P. O. Ludl, S. Morisi and E. Peinado, The Reactor mixing angle and CP violation with two texture zeros in the light of T2K, Nucl. Phys. B 857, 411 (2012) [arXiv:1109.3393 [hep-ph]]. 
[42] X. -G. He and A. Zee, Minimal modification to the tri-bimaximal neutrino mixing, Phys. Lett. B 645, 427 (2007) [hep-ph/0607163].

[43] S. F. Ge, H. J. He and F. R. Yin, JCAP 1005, 017 (2010) [arXiv:1001.0940 [hep-ph]].

[44] H. J. He and F. R. Yin, Phys. Rev. D 84, 033009 (2011) [arXiv:1104.2654 [hep-ph]].

[45] X. -G. He and A. Zee, Minimal Modification to Tri-bimaximal Mixing, Phys. Rev. D 84, 053004 (2011) [arXiv:1106.4359 [hep-ph]].

[46] J. Liao, D. Marfatia and K. Whisnant, Phys. Rev. D 87, no. 1, 013003 (2013) doi:10.1103/PhysRevD.87.013003 [arXiv:1205.6860 [hep-ph]].

[47] R. Dutta, U. Ch, A. K. Giri and N. Sahu, Perturbative Bottom-up Approach for Neutrino Mass Matrix in Light of Large $\theta_{13}$ and Role of Lightest Neutrino Mass, arXiv:1303.3357 [hep-ph].

[48] D. V. Ahluwalia, CP violating Tri-bimaximal-Cabibbo mixing, ISRN High Energy Phys. 2012, 954272 (2012) [arXiv:1206.4779 [hep-ph]].

[49] Y. Shimizu, R. Takahashi and M. Tanimoto, Minimal Neutrino Texture with Neutrino Mass Ratio and Cabibbo Angle, PTEP 2013, no. 6, 063 B02 (2013) [arXiv:1212.5913 [hep-ph]].

[50] D. Borah, arXiv:1307.2426 [hep-ph].

[51] W. Rodejohann and H. Zhang, arXiv:1402.2226 [hep-ph].

[52] D. Zhuridov, arXiv:1304.4870 [hep-ph].

[53] J. Kile, M. J. PÃl'rez, P. Ramond and J. Zhang, Phys. Rev. D 90, no. 1, 013004 (2014) [arXiv:1403.6136 [hep-ph]].

[54] D. Zhuridov, arXiv:1405.5522 [hep-ph].

[55] S. T. Petcov, Nucl. Phys. B 892, 400 (2015) [arXiv:1405.6006 [hep-ph]].

[56] S. K. Kang and C. S. Kim, Phys. Rev. D 90, no. 7, 077301 (2014) [arXiv:1406.5014 [hep-ph]].

[57] I. Girardi, S. T. Petcov and A. V. Titov, Nucl. Phys. B 894, 733 (2015) [arXiv:1410.8056 [hep-ph]].

[58] A. Damanik, arXiv:1505.00681 [hep-ph]. 
[59] Z. z. Xing and Z. h. Zhao, Rept. Prog. Phys. 79, no. 7, 076201 (2016) [arXiv:1512.04207 [hep-ph]].

[60] J. Zhang and S. Zhou, JHEP 1609, 167 (2016) [arXiv:1606.09591 [hep-ph]].

[61] R. Samanta and M. Chakraborty, arXiv:1802.04751 [hep-ph].

[62] Z. h. Zhao, arXiv:1803.04603 [hep-ph].

[63] P. Chen, S. Centelles ChuliÃą, G. J. Ding, R. Srivastava and J. W. F. Valle, arXiv:1806.03367 [hep-ph].

[64] Y. Shimizu, M. Tanimoto and A. Watanabe, Breaking Tri-bimaximal Mixing and Large ө13, Prog. Theor. Phys. 126, 81 (2011) [arXiv:1105.2929 [hep-ph]].

[65] A. S. Joshipura and K. M. Patel, Viability of the exact tri-bimaximal mixing at $M_{G U T}$ in SO(10), JHEP 1109, 137 (2011) [arXiv:1105.5943 [hep-ph]].

[66] S. Morisi, K. M. Patel and E. Peinado, Model for T2K indication with maximal atmospheric angle and tri-maximal solar angle, Phys. Rev. D 84, 053002 (2011) [arXiv:1107.0696 [hep-ph]].

[67] P. S. Bhupal Dev, R. N. Mohapatra and M. Severson, Neutrino Mixings in SO(10) with Type II Seesaw and $\theta_{13}$, Phys. Rev. D 84, 053005 (2011) [arXiv:1107.2378 [hep-ph]].

[68] R. d. A. Toorop, F. Feruglio and C. Hagedorn, Discrete Flavour Symmetries in Light of T2K, Phys. Lett. B 703, 447 (2011) [arXiv:1107.3486 [hep-ph]].

[69] A. Adulpravitchai and R. Takahashi, A4 Flavor Models in Split Seesaw Mechanism, JHEP 1109, 127 (2011) [arXiv:1107.3829 [hep-ph]].

[70] A. Rashed and A. Datta, The charged lepton mass matrix and non-zero $\theta_{13}$ with TeV scale New Physics, Phys. Rev. D 85, 035019 (2012) [arXiv:1109.2320 [hep-ph]].

[71] S. Gupta, A. S. Joshipura and K. M. Patel, Minimal extension of tri-bimaximal mixing and generalized $Z_{2} X Z_{2}$ symmetries, Phys. Rev. D 85, 031903 (2012) [arXiv:1112.6113 [hep-ph]].

[72] S. F. King and C. Luhn, A4 models of tri-bimaximal-reactor mixing, JHEP 1203, 036 (2012) [arXiv:1112.1959 [hep-ph]].

[73] P. S. Bhupal Dev, B. Dutta, R. N. Mohapatra and M. Severson, $\theta_{13}$ and Proton Decay in a Minimal $S O(10) \times S_{4}$ model of Flavor, Phys. Rev. D 86, 035002 (2012) [arXiv:1202.4012 [hep-ph]]. 
[74] J. C. GÃşmez-Izquierdo and M. MondragÃşs, arXiv:1804.08746 [hep-ph].

[75] M. H. Rahat, P. Ramond and B. Xu, Phys. Rev. D 98, no. 5, 055030 (2018) [arXiv:1805.10684 [hep-ph]].

[76] K. S. Babu, E. Ma and J. W. F. Valle, Underlying A(4) symmetry for the neutrino mass matrix and the quark mixing matrix, Phys. Lett. B 552, 207 (2003) [hep-ph/0206292].

[77] A. Zee, Obtaining the neutrino mixing matrix with the tetrahedral group, Phys. Lett. B 630, 58 (2005) [hep-ph/0508278].

[78] E. Ma, Tetrahedral family symmetry and the neutrino mixing matrix, Mod. Phys. Lett. A 20, 2601 (2005) [hep-ph/0508099].

[79] B. Adhikary, B. Brahmachari, A. Ghosal, E. Ma and M. K. Parida, A(4) symmetry and prediction of U(e3) in a modified Altarelli-Feruglio model, Phys. Lett. B 638, 345 (2006) [hep-ph/0603059].

[80] G. Altarelli, F. Feruglio and L. Merlo, Tri-Bimaximal Neutrino Mixing and Discrete Flavour Symmetries, arXiv:1205.5133 [hep-ph].

[81] G. Altarelli and F. Feruglio, Discrete Flavor Symmetries and Models of Neutrino Mixing, Rev. Mod. Phys. 82, 2701 (2010) [arXiv:1002.0211 [hep-ph]].

[82] H. Ishimori, T. Kobayashi, H. Ohki, Y. Shimizu, H. Okada and M. Tanimoto, NonAbelian Discrete Symmetries in Particle Physics, Prog. Theor. Phys. Suppl. 183, 1 (2010) [arXiv:1003.3552 [hep-th]].

[83] D. Hernandez and A. Y. Smirnov, Phys. Rev. D 87, no. 5, 053005 (2013) [arXiv:1212.2149 [hep-ph]].

[84] S. F. King and C. Luhn, Neutrino Mass and Mixing with Discrete Symmetry, Rept. Prog. Phys. 76, 056201 (2013) [arXiv:1301.1340 [hep-ph]].

[85] E. Ma, Neutrino mass matrix from S(4) symmetry, Phys. Lett. B 632, 352 (2006) [hep$\mathrm{ph} / 0508231]$.

[86] C. S. Lam, The Unique Horizontal Symmetry of Leptons, Phys. Rev. D 78, 073015 (2008) [arXiv:0809.1185 [hep-ph]].

[87] C. S. Lam, A Horizontal Symmetry for Leptons and Quarks, arXiv:1105.4622 [hep-ph].

[88] G. Altarelli and F. Feruglio, Discrete Flavor Symmetries and Models of Neutrino Mixing, Rev. Mod. Phys. 82, 2701 (2010) [arXiv:1002.0211 [hep-ph]]. 
[89] J. C. GÃß̧mez-Izquierdo and M. MondragÃşn, arXiv:1804.08746 [hep-ph].

[90] X. -G. He and A. Zee, Minimal modification to the tri-bimaximal neutrino mixing, Phys. Lett. B 645, 427 (2007) [hep-ph/0607163].

[91] C. H. Albright and W. Rodejohann, Comparing Trimaximal Mixing and Its Variants with Deviations from Tri-bimaximal Mixing, Eur. Phys. J. C 62, 599 (2009) [arXiv:0812.0436 [hep-ph]].

[92] Z. -Z. Xing, The T2K Indication of Relatively Large $\theta_{13}$ and a Natural Perturbation to the Democratic Neutrino Mixing Pattern, Chin. Phys. C 36, 101 (2012) [arXiv:1106.3244 [hep-ph]].

[93] X. -G. He and A. Zee, Minimal Modification to Tri-bimaximal Mixing, Phys. Rev. D 84, 053004 (2011) [arXiv:1106.4359 [hep-ph]].

[94] W. Chao and Y. -j. Zheng, Relatively Large Theta13 from Modification to the Tribimaximal, Bimaximal and Democratic Neutrino Mixing Matrices, JHEP 1302, 044 (2013) [arXiv:1107.0738 [hep-ph]].

[95] Z. h. Zhao, JHEP 1411, 143 (2014) [arXiv:1405.3022 [hep-ph]].

[96] S. K. Garg, Int. J. Mod. Phys. A 34, no. 01, 1950005 (2019) [arXiv:1806.06658 [hep-ph]].

[97] S. K. Garg and S. Gupta, JHEP 1310, 128 (2013)[arXiv:1308.3054 [hep-ph]].

[98] S. K. Garg, Nucl. Phys. B 931, 469 (2018) [arXiv:1712.02212 [hep-ph]].

[99] B. Wang, J. Tang and X. Q. Li, Phys. Rev. D 88, 073003 (2013) [arXiv:1303.1592 [hep-ph]].

[100] I. Girardi, S. T. Petcov and A. V. Titov, Eur. Phys. J. C 75, 345 (2015) [arXiv:1504.00658 [hep-ph]].

[101] G. Altarelli, F. Feruglio and I. Masina, Can neutrino mixings arise from the charged lepton sector?, Nucl. Phys. B 689, 157 (2004) [hep-ph/0402155].

[102] A. Romanino, Charged lepton contributions to the solar neutrino mixing and theta(13), Phys. Rev. D 70, 013003 (2004) [hep-ph/0402258].

[103] S. Antusch and S. F. King, Charged lepton corrections to neutrino mixing angles and CP phases revisited, Phys. Lett. B 631, 42 (2005) [hep-ph/0508044].

[104] J. C. Gomez-Izquierdo and A. Perez-Lorenzana, Phys. Rev. D 77, 113015 (2008) [arXiv:0711.0045 [hep-ph]]. 
[105] J. C. Gomez-Izquierdo and A. Perez-Lorenzana, Phys. Rev. D 82, 033008 (2010) [arXiv:0912.5210 [hep-ph]].

[106] D. Marzocca, S. T. Petcov, A. Romanino and M. Spinrath, Sizeable $\theta_{13}$ from the Charged Lepton Sector in SU(5), (Tri-)Bimaximal Neutrino Mixing and Dirac CP Violation, JHEP 1111, 009 (2011) [arXiv:1108.0614 [hep-ph]].

[107] S. Dev, S. Gupta and R. R. Gautam, Parametrizing the Lepton Mixing Matrix in terms of Charged Lepton Corrections, Phys. Lett. B 704, 527 (2011) [arXiv:1107.1125 [hep-ph]].

[108] D. Marzocca, S. T. Petcov, A. Romanino and M. C. Sevilla, Nonzero $\left|U_{e} 3\right|$ from Charged Lepton Corrections and the Atmospheric Neutrino Mixing Angle, JHEP 1305, 073 (2013) [arXiv:1302.0423 [hep-ph]].

[109] D. Zhuridov, arXiv:1304.4870 [hep-ph].

[110] J. C. GÂşmez-Izquierdo, Eur. Phys. J. C 77, no. 8, 551 (2017) [arXiv:1701.01747 [hep-ph]].

[111] J. C. GÃ̧̧mez-Izquierdo, F. Gonzalez-Canales and M. MondragÂAş, Int. J. Mod. Phys. A 32, no. 28-29, 1750171 (2017) [arXiv:1705.06324 [hep-ph]].

[112] H. Fritzsch and Z. -Z. Xing, Phys. Lett. B 372, 265 (1996) [hep-ph/9509389].

[113] H. Fritzsch and Z. -z. Xing, Phys. Lett. B 440, 313 (1998) [hep-ph/9808272].

[114] M. Fukugita, M. Tanimoto and T. Yanagida, Phys. Rev. D 57, 4429 (1998) [hep$\mathrm{ph} / 9709388]$.

[115] W. Rodejohann and Z. -z. Xing, Phys. Lett. B 601, 176 (2004) [hep-ph/0408195].

[116] S. Pakvasa, W. Rodejohann and T. J. Weiler, Unitary parametrization of perturbations to tribimaximal neutrino mixing, Phys. Rev. Lett. 100, 111801 (2008) [arXiv:0711.0052 [hep-ph]].

[117] T. Kitabayashi and M. Yasue, Phys. Lett. B 713 (2012) 206 [arXiv:1204.4523 [hep-ph]].

[118] S. M. Boucenna, S. Morisi, M. Tortola and J. W. F. Valle, Bi-large neutrino mixing and the Cabibbo angle, Phys. Rev. D 86, 051301 (2012) [arXiv:1206.2555 [hep-ph]].

[119] B. Hu, Neutrino Mixing: Perturbation, Parameterization and Trimaximal-Cabibbo Mixing, Phys. Rev. D 87, 053011 (2013) arXiv:1212.4079 [hep-ph]. 